\title{
A general method of estimating stellar astrophysical parameters from photometry
}

\author{
A. N. Belikov ${ }^{1,2}$ and S. Röser ${ }^{2}$ \\ ${ }^{1}$ Kapteyn Astronomical Institute, PO Box 800, 9700 AV Groningen, The Netherlands \\ e-mail: A.N.Belikov@astro.rug.nl \\ 2 Astronomisches Rechen-Institut, Mönchhofstrasse 12-14, 69120 Heidelberg, Germany \\ e-mail: roeser@ari.uni-heidelberg.de
}

Received 19 December 2007 / Accepted 17 July 2008

\begin{abstract}
Context. Applying photometric catalogs to the study of the population of the Galaxy is obscured by the impossibility to map directly photometric colors into astrophysical parameters. Most of all-sky catalogs like ASCC or 2MASS are based upon broad-band photometric systems, and the use of broad photometric bands complicates the determination of the astrophysical parameters for individual stars.

Aims. This paper presents an algorithm for determining stellar astrophysical parameters (effective temperature, gravity and metallicity) from broad-band photometry even in the presence of interstellar reddening. This method suits the combination of narrow bands as well.

Methods. We applied the method of interval-cluster analysis to finding stellar astrophysical parameters based on the newest Kurucz models calibrated with the use of a compiled catalog of stellar parameters.

Results. Our new method of determining astrophysical parameters allows all possible solutions to be located in the effective temperature-gravity-metallicity space for the star and selection of the most probable solution.
\end{abstract}

Key words. methods: data analysis - techniques: photometric - stars: fundamental parameters

\section{Introduction}

Estimating the astrophysical parameters of stars is one of the core problems in astrophysics and the key solving a number of astrophysical questions. Indeed, the knowledge of effective temperature, luminosity class (or gravity at the surface of the star), and metallicity of the star allows the distance to the star, the age of the star, and the extinction in the interstellar medium between the star and the observer to be estimated.

The number of stars with detailed spectral information is very limited compared to the wealth of data in photometry. The problem of determining astrophysical parameters with photometry is usually solved with the help of the narrow-band photometric systems (for instance, the Strömgren photometric system) that provide quite good precision for a temperature, gravity, and metallicity. For example, Nordström (2004) estimates the metallicity from Strömgren photometry with the final average 0.12 dex dispersion around the spectroscopic values. The problem becomes much more complicated in the case of broad-band photometry where all fine features in the photometric bands (tracers of the differences in metallicity and especially in gravity) are effectively smoothed. The estimation of $T_{\text {eff }}, \log g$, and $[\mathrm{M} / \mathrm{H}]$ is crucial for the study of the Galaxy on the basis of broad-band all-sky surveys like 2MASS or the broad-band deep surveys like SDSS/SEGUE or UKIDSS.

The history of stellar parameter determination from broadband photometry was started by Johnson in the middle of the $50 \mathrm{~s}$ of the last century (see, for example, Johnson 1953, where the author defined extinction-free colors for the $U B V$ photometric system). Later, some broad-band photometric systems showed the usefulness of extinction-free colors for determining of temperatures and gravities with some assumptions about the metallicity (for example, the Vilnus photometric system). The use of extinction-free colors is discussed in detail in Straižys (1977).

A major problem of using extinction-free colors is how to determine extinction-free colors themselves from the observed ones. Indeed, as we show below, to calculate the extinction-free indices and to use them for the estimation of astrophysical parameters we have to accept some initial guesses about the estimated astrophysical parameters of the star a priori.

In this study we minimize the amount of information to be assumed a priori for the estimation of astrophysical parameters of the star. We will not make guesses about the astrophysical parameters of the star (temperature, gravity or metallicity) based on the position of the star, e.g. relative to the galactic plane.

We do not discuss the dependence of the estimated parameter on the synthetic model grid adopted in this study and do not compare different synthetic model grids. The estimated parameters will always depend on the consistency of the synthetic models used in the study. However, in the case of Johnson $B, V, J, H$, and $K_{\mathrm{S}}$ photometry, we calibrated the parameters obtained from the model fluxes by our method with published parameters from different authors.

\section{The synthetic grid of magnitudes and colors}

To start with the determination of the astrophysical parameters from photometry we have to provide a synthetic grid fine enough to give us synthetic magnitudes and colors for each point of the grid. 


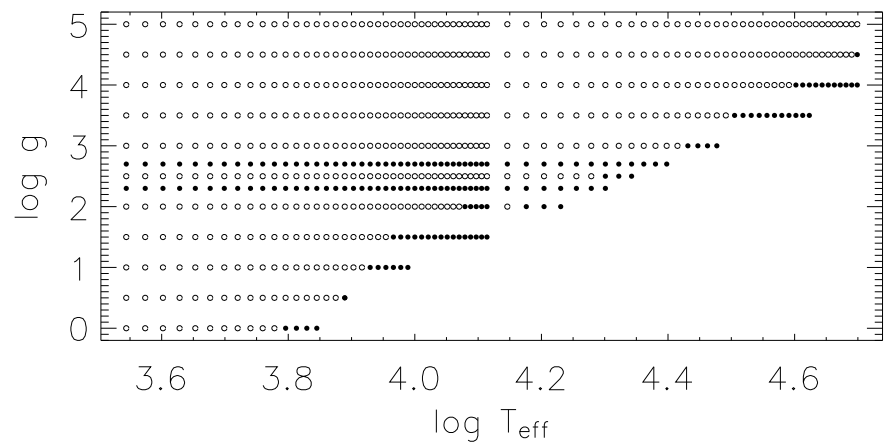

Fig. 1. The synthetic grid of stellar atmospheres used in the study. Open circles are original models calculated by Castelli (see Castelli \& Kurucz 2003) and the closed circles are models calculated by us with the use of ATLAS9 program to fill the gap in the original grid.

In this section we describe the definition of terms used in the paper, the computation of the synthetic grid of colors and magnitudes, the influence of extinction and the definition of the extinction-free colors. We create a grid of synthetic colors, use an assumed extinction law (Cardelli et al. 1989) to find reddened colors and to construct finally a grid of a synthetic extinctionfree indices.

\subsection{Definition of synthetic magnitudes}

The definition of synthetic magnitudes depends on the type of detector used in the observation that we are going to approximate. If the detected flux corrected for the atmospheric extinction is $f_{\lambda}$ and the stellar flux at the surface of the star is $F_{\lambda}$ (this is the flux delivered by synthetic atmosphere models), they are related to each other as

$f_{\lambda}=10^{-0.4 A_{\lambda}}(R / d)^{2} F_{\lambda}$

where $A_{\lambda}$ is the interstellar extinction at the wavelength $\lambda, R$ the stellar radius, and $d$ the distance to the star, $R$ and $d$ are in the same units. In the case of photon-counting devices the magnitude of the star $m_{S}$ in the filter $S_{\lambda}$ is

$m_{S}=-2.5 \log \frac{\int_{\lambda_{1}}^{\lambda_{2}} \lambda f_{\lambda} S_{\lambda} \mathrm{d} \lambda}{\int_{\lambda_{1}}^{\lambda_{2}} \lambda S_{\lambda} \mathrm{d} \lambda}+m_{S}^{0}$.

We discuss the zero-points of photometric systems $m_{S}^{0}$ in Sect. 3 .

\subsection{Theoretical models used in the study}

We used the latest Kurucz models of theoretical spectra (Castelli \& Kurucz 2003), because these models can provide wider coverage in parameter space compared to other libraries. The grid used in the study consists of models with temperatures from $50000 \mathrm{~K}$ to $3500 \mathrm{~K}, \log g$ from 0.0 to 5.0 , and metallicities from $[\mathrm{M} / \mathrm{H}]=-2.5$ to 0.5 . We extended this initial grid of models to lower gravity and added two points on the gravity axis ( $\log g=2.3$ and $\log g=2.7$ ) using the program ATLAS9 by Castelli \& Kurucz (2003). The final grid of original models and newly calculated models is presented in Fig. 1. These models do not have overshooting, but do have microturbulent velocity $\xi=2 \mathrm{~km} \mathrm{~s}^{-1}$. The grid was interpolated with cubic splines to provide a step in $\Delta \log T_{\text {eff }}=0.005 \mathrm{dex}$, a step $\Delta \log g=0.1 \mathrm{dex}$, and a step $\Delta[\mathrm{M} / \mathrm{H}]=0.1$ dex.

To calculate absolute magnitudes from Kurucz models, we had to adopt stellar radii for each point in the $T_{\text {eff }}-\log g$ grid.

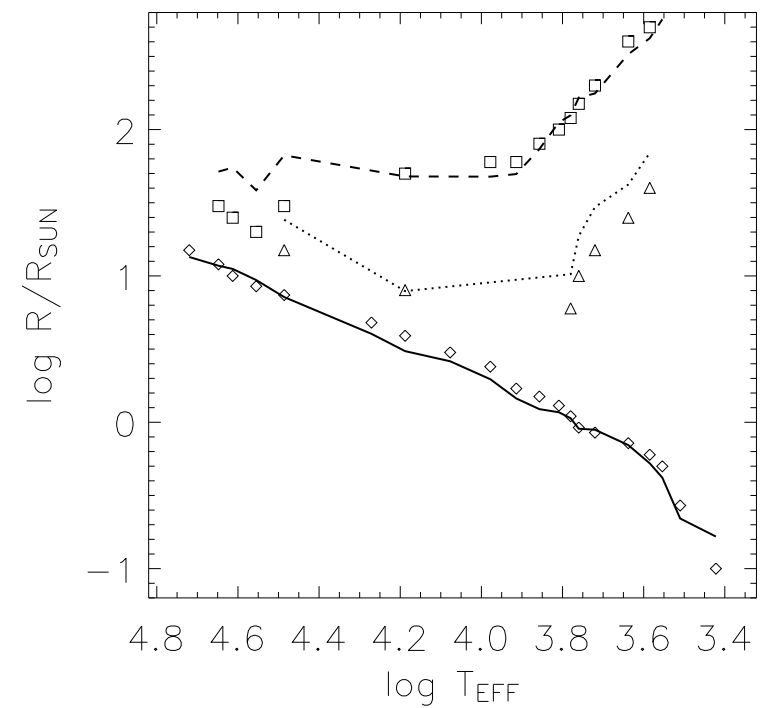

Fig. 2. Comparison of radii used in this study with the radii for the main sequence (diamonds), giants (triangles), and supergiants (squares) from Schmidt-Kaler (1982). The solid curve is the theoretical radiustemperature dependence for the main sequence, the dotted curve the theoretical radius-temperature dependence for giants, and the dashed curve the theoretical radius-temperature dependence for supergiants.

We took radii from the theoretical track systems of Palla \& Stahler (1993), D’Antona \& Mazzitelli (1994) for pre-main sequence stars, and Geneva tracks (Schaller et al. 1992) for postmain sequence stars. Figure 2 shows the agreement between the radii from the compiled track system (points) and the "standard" radii for main sequence stars, giants, and supergiants from Table 23 of Landolt-Börnstein (Schmidt-Kaler 1982). The coincidence is generally quite satisfactory except in the case of hot $\left(\log T_{\text {eff }} \geq 4.3\right.$ ) supergiants.

We used the following sets of filters in the study: Johnson $B$ and $V$ filters from the UBVBUSER code (Buser 1978) and 2MASS $J, H$, and $K_{\mathrm{S}}$ filters (Cutri et al. 2006).

\subsection{Extinction in the case of a broad-band photometric system}

To compute a set of extinction-free indices, we have to compute first reddened magnitudes and colors for a wide range of interstellar extinction. This gives us synthetic color excesses for each model in the grid. We adopt the extinction law $A_{\lambda}$ from Cardelli et al. (1989), and take $A_{V}=3.11 E_{B-V}$, computing a grid of models with the step in extinction $\Delta A_{V}=0.05 \mathrm{mag}$.

The extinction law used in the study (Cardelli et al. 1989) was constructed with the use of $\mathrm{O}$ - and early B-stars. This extinction law is a polynomial approximation of the extinction for the wavelength $\lambda\left\langle A_{\lambda} / A_{V}\right\rangle=a(1 / \lambda)+b(1 / \lambda) / R_{V}$. In fact, this value of $R_{V}$ is a parameter related to OB stars. This also means that extinction constructed with the use of an extinction law $A_{\lambda} / A_{V}$ will give different values of the extinction for different spectral classes in the same photometric band and with the same distance and ISM between star and observer. Let us estimate this difference in extinction in the Johnson $V$ band depending on the spectral class and luminosity class of the star. We calculate the extinction as the difference between theoretical magnitudes for the Johnson $V$ filter and the stellar atmosphere model with 


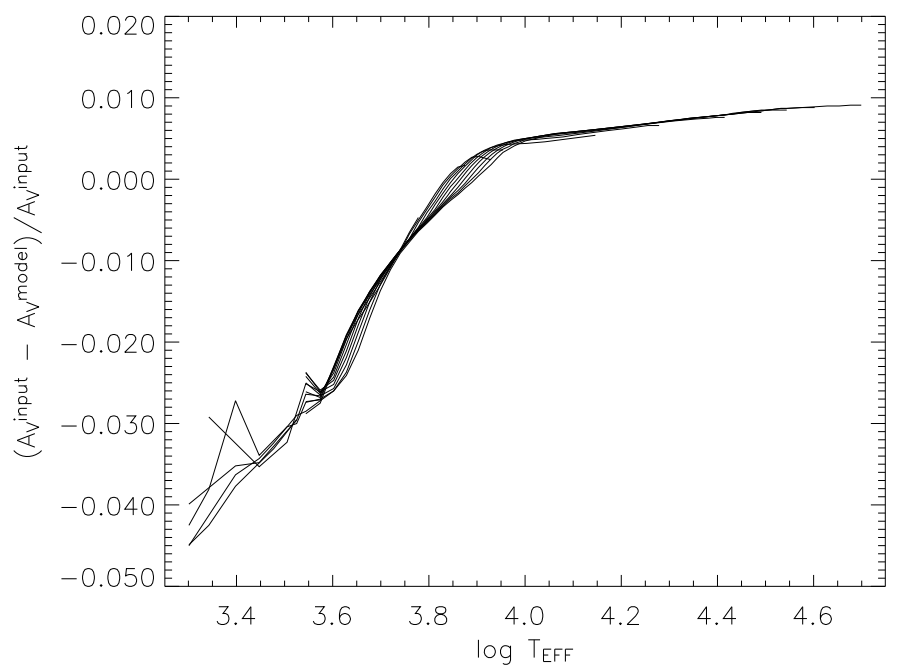

Fig. 3. Differences in the extinction law. Solid lines are the differences in true and nominal extinction in the Johnson $V$ band for stars of different temperatures for different gravities (from $\log g 0.0$ to 5.0 with 0.5 dex step size). Solar metallicity is assumed.

$\log T_{\text {eff }}, \log g,[\mathrm{M} / \mathrm{H}]$ as $A_{V}^{\text {model }}=m_{V}\left(A_{V}^{\text {input }}\right)-m_{V}\left(A_{V}^{\text {input }}=0\right)$ with the use of $A_{\lambda}\left(A_{V}\right)$ from Cardelli et al. (1989):

$A_{V}^{\text {model }}=-2.5 \log \frac{\int_{\lambda_{1}}^{\lambda_{2}} 10^{-0.4 A_{\lambda}\left(A_{V}^{\text {input }}\right)} \lambda f_{\lambda} S_{\lambda} \mathrm{d} \lambda}{\int_{\lambda_{1}}^{\lambda_{2}} \lambda f_{\lambda} S_{\lambda} \mathrm{d} \lambda}$.

The output $A_{V}^{\text {model }}$ differs from the input $A_{V}^{\text {input }}$ and this difference depends on the spectral class, gravity, and metallicity of the star. This difference is a physical phenomenon. Indeed, an O5 star and an M 5 star being placed at the same distance from the observer and with the same optical depth of the ISM will be differently obscured by the ISM. This difference reaches up to $5 \%$ of the nominal value of an extinction for the later spectral types.

\subsection{Extinction-free color indices}

Extinction-free color indices (or $Q$-values) are the final target for our computations of synthetic colors. With the use of these indices, we are able to reduce the number of models in our grid, because we can use a unique grid of synthetic models for any star independent of the individual extinction for this star.

The construction of extinction-free color indices has some problems that we consider below. The $Q$-values are by definition

$$
\begin{aligned}
Q_{m_{1} m_{2} m_{3}} & =\left(m_{1}-m_{2}\right)_{0}-\frac{E_{m_{1} m_{2}}}{E_{m_{2} m_{3}}}\left(m_{2}-m_{3}\right)_{0} \\
& =\left(m_{1}-m_{2}\right)-\frac{E_{m_{1} m_{2}}}{E_{m_{2} m_{3}}}\left(m_{2}-m_{3}\right) .
\end{aligned}
$$

However, $E_{m_{1} m_{2}} / E_{m_{2} m_{3}}$ is not constant, but depends on the spectral class, gravity and metallicity of star (see, for example, Grebel \& Roberts 1995). The excess ratio depends as well on the extinction itself. By definition, $E_{m_{1} m_{2}}=A_{m_{1}}-A_{m_{2}}$, so to remove extinction from the excess ratio, we have to replace $A_{m_{1}}$ with $A_{m_{2}}$ or vice versa. However, the dependence of the extinction on the optical depth is not linear (see, for instance, Chap. 6.5 from Straižys 1977). As a result, we have a dependence $A_{m_{1}}=k_{1} A_{m_{2}}+k_{2} A_{m_{2}}^{2}+\ldots$, and to construct an excess ratio, we have to neglect orders higher than the first in this dependence. For the precise determination of astrophysical parameters, we have to check the possibility of neglecting these higher

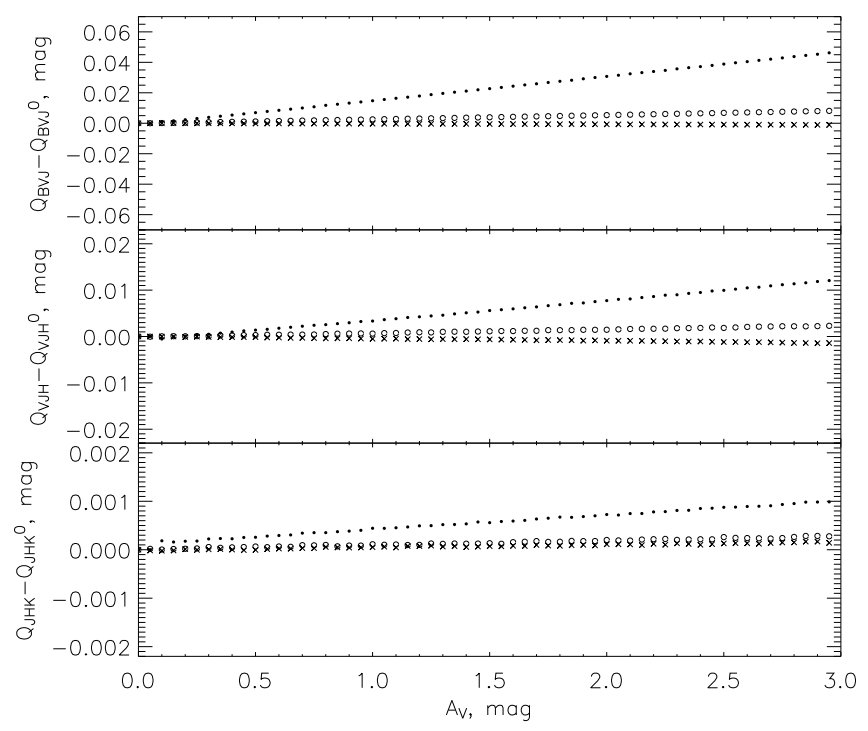

Fig. 4. Dependence of the $Q$ values on $A_{V}$ for stars with solar metallicities, $\log g=4.5$, and $T_{\text {eff }}=3500 \mathrm{~K}$ (filled circles), $T_{\text {eff }}=7000 \mathrm{~K}$ (open circles), and $T_{\text {eff }}=10000 \mathrm{~K}$ (crosses).

order coefficients. Figure 4 shows the remaining dependence of the $Q$-values on extinction, which can indeed be neglected compared to the $Q$-values themselves in Fig. 5.

\section{Calibration of theoretical models}

As we see from Eq. (2), we have to bring our theoretical models into accordance with the zeropoint of the photometric system $\left(m_{S}^{0}\right)$.

To provide this calibration, we compiled a catalog of stars with temperature, gravity, and metallicity known from spectroscopy. The catalog is a combination from the catalogs of Borkova \& Marsakov (2005) and Cayrel de Strobel et al. (1997) with the addition of the catalogs of Soubiran et al. (1998), Erspamer \& North (2003) and Takeda et al. (2005). Our final list contains 3092 stars, 2096 of them were identified with the ASCC catalog (Kharchenko 2001). The latter contains 2501304 stars with Johnson $B, V$ photometry recalculated from the Tycho $B_{\mathrm{T}}$, $V_{\mathrm{T}}$ and parallaxes and proper motions (Hipparcos and Tycho-1). We crossmatched the ASCC catalog with the 2MASS catalog, so that the stars in our compilation (2096 stars which we will call "calibration catalog" further on) have Johnson $B, V$ and 2MASS $J, H, K_{\mathrm{s}}$ magnitudes.

A subsample of 1287 stars was selected with $\sigma_{B}<0.1 \mathrm{mag}$, $\sigma_{V}<0.1 \mathrm{mag}, \sigma_{J}<0.3 \mathrm{mag}, \sigma_{H}<0.3 \mathrm{mag}, \sigma_{K_{\mathrm{s}}}<$ $0.3 \mathrm{mag}$, parallaxes with $\sigma_{\pi} / \pi$ better than $10 \%$ and distances within $100 \mathrm{pc}$. We assume that the stars from this subsample are extinction-free and use the subsample to calibrate our theoretical models.

To calibrate the theoretical sequences, we selected all stars from the subsample with $\log g=4.0 \pm 0.1,[\mathrm{M} / \mathrm{H}]=0.0 \pm 0.2$, and photometric errors in all bands better than 0.05 mag and absolute magnitudes in the $B$ band $M_{B}<6.0 \mathrm{mag}$.

On the basis of this calibration subsample of 58 selected stars, we calibrated the synthetic models

$$
\begin{aligned}
(B-V)^{C} & =(B-V)^{T}+(0.6001 \pm 0.0445) \\
(V-J)^{C} & =(V-J)^{T}+(2.6764 \pm 0.0896) \\
(J-H)^{C} & =(J-H)^{T}+(1.0382 \pm 0.0361) \\
\left(H-K_{\mathrm{S}}\right)^{C} & =\left(H-K_{\mathrm{S}}\right)^{T}+(1.1141 \pm 0.0290) .
\end{aligned}
$$




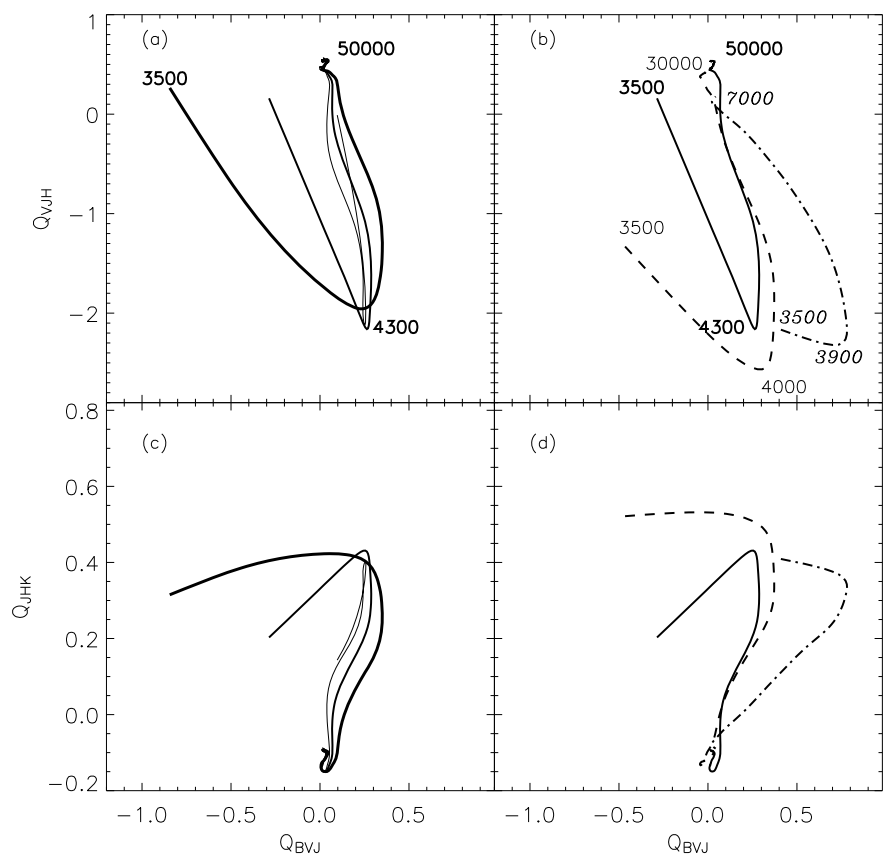

Fig. 5. The $Q$ diagrams. Panel a) shows the $Q_{B V J}-Q_{V J H}$ diagram for 3 different metallicities (the thick line is for $[\mathrm{M} / \mathrm{H}]=0.5$, the intermediate line for $[\mathrm{M} / \mathrm{H}]=0.0$, and the thin line for $[\mathrm{M} / \mathrm{H}]=-0.5)$, and $\log g=4.5$. Effective temperatures for $[\mathrm{M} / \mathrm{H}]=0.5$ are shown, effective temperatures for other metallicities are, respectively, $50000 \mathrm{~K}$ for the point with maximum $Q_{V J H}, 4300 \mathrm{~K}$ for the point with minimum $Q_{V J H}$, and $3500 \mathrm{~K}$ for the end point of the sequence. Panel b) is the $Q_{B V J}-Q_{V J H}$ diagram for solar metallicity and $\log g 0.0$ (dotted-dashed line), 3.0 (dashed line), and 4.5 (solid line). Effective temperatures are shown for $\log g=0.0$ (in italics, $7000 \mathrm{~K}$ for the point with maximum $Q_{V J H}, 3900 \mathrm{~K}$ for the point with maximum $Q_{B V J}$ and $3500 \mathrm{~K}$ for the end point of the sequence), $\log g=3.0(30000 \mathrm{~K}$ for the point with maximum $Q_{V J H}, 4000 \mathrm{~K}$ for the point with minimum $Q_{V J H}$, and $3500 \mathrm{~K}$ for the end point of the sequence), $\log g=4.5$ (in bold, $50000 \mathrm{~K}$ for the point with maximum $Q_{V J H}, 4300 \mathrm{~K}$ for the point with minimum $Q_{V J H}$, and $3500 \mathrm{~K}$ for the end point of the sequence). Panels c) and d) are the same but for $Q_{B V J}-Q_{J H K_{\mathrm{S}}}$ diagram.

Although, the parameters from literature cover only a small fraction of the full parameter space, we applied the corrections to the full sythetic grid. Comparison of our calibrated colors with the colors for Vega shows good agreement. We take the atmospheric parameters for Vega from Smith \& Dworetsky (1993) $\left(T_{\text {eff }}=9450 \mathrm{~K}, \log g=4.00,[\mathrm{M} / \mathrm{H}]=-0.55\right)$, which from our calibrated model grid yields $(B-V)=0.005$ mag, $(V-J)=$ $0.014 \mathrm{mag},(J-H)=-0.060 \mathrm{mag},\left(H-K_{\mathrm{s}}\right)=0.052 \mathrm{mag}$. The 2MASS $J H K_{\mathrm{S}}$ photometric system differs slightly from the Johnson JHK (see Carpenter 2001; Koen et al. 2007).

\section{The interval-cluster method of the astrophysical parameters estimation}

Parameters like effective temperature, gravity, metallicity, extinction, and distance on the basis of broad-band photometry must be determined directly from photometry without any a priori assumption about physical properties of stars that can restrict the range of the search. Suppose that we have a parameter space $\left\{X_{0}\right\}=\left\{T_{\text {eff }}, \log g,[\mathrm{M} / \mathrm{H}], A_{V}, r\right\}$, where $T_{\text {eff }}$ is the effective temperature, $\log g$ the logarithm of gravity, $[\mathrm{M} / \mathrm{H}]$ the metallicity, $A_{V}$ the extinction in the Johnson $V$ band ( $A_{V}$ is used as the parameter for extinction hereafter), and $r$ the distance to the star. From observations (or from a synthetic grid in the case

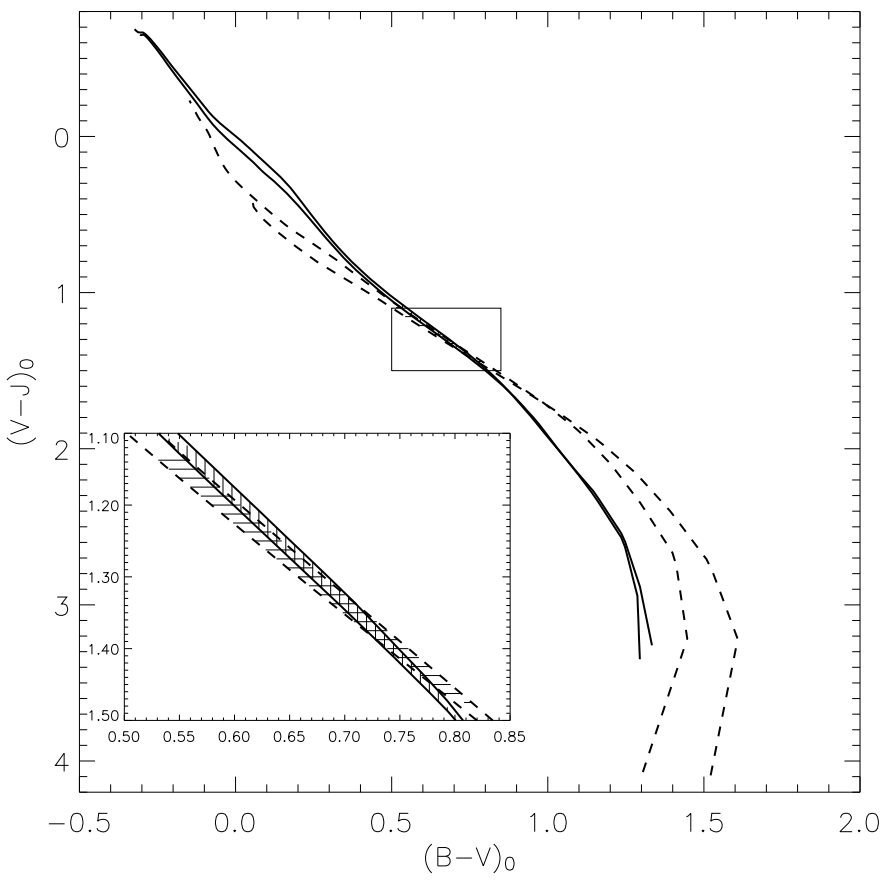

Fig. 6. The color-color diagram for $(B-V)_{0}$ and $(V-J)_{0}$ colors. Solid lines are for metallicity $[\mathrm{M} / \mathrm{H}]=-0.5$ and $\log g$ between 4.5 and 5.0, dashed lines for solar metallicity and $\log g$ between 1.0 and 2.0.

of simulations), we have a magnitude space $\left\{Y_{0}\right\}=\left\{m_{1}, \ldots, m_{n}\right\}$ that can be transformed to the color space $\{Y\}=\left\{m_{i}-m_{j}, i \neq j\right\}$. Via this transformation, we eliminate the distance $r$ from the parameter space $\left\{X_{0}\right\}$ and work in the parameter space $\{X\}=\left\{T_{\text {eff }}\right.$, $\left.\log g,[\mathrm{M} / \mathrm{H}], A_{V}\right\}$.

Let us suppose that there are two different subsets in the parameter space $\left\{X^{1}\right\}$ and $\left\{X^{2}\right\}$. These two classes can be populated by stars within any given temperature range, gravity range, and metallicity range. Let us as well have two subsets in the color space $\left\{Y^{1}\right\}$ and $\left\{Y^{2}\right\}$ containing the synthetic colors of the elements in $\left\{X^{1}\right\}$ and $\left\{X^{2}\right\}$. The task of classification is to attribute the star with some colors to one of the classes in the parameter space $\{X\}$. This is possible if the two subsets $\left\{Y^{1}\right\}$ and $\left\{Y^{2}\right\}$ are separated in the color space, but this requirement is not always guaranteed in the case of broad-band photometry. Stars with different temperatures, gravities, metallicities, and extinction can occupy the same area in the color space.

Figure 6 illustrates the problem: stars within two different metallicities and gravities $([\mathrm{M} / \mathrm{H}]=-0.5, \log g \in[4.5,5.0]$ and $[\mathrm{M} / \mathrm{H}]=0.0, \log g \in[1.0,2.0])$ occupy the same space in the 2 -color diagram even in the absence of extinction. It is obvious that for this range of colors there are at least two different solutions in parameter space for the star inside the filled area.

In this case, there are at least two possible solutions for the parameters of a star: $\left\{X^{1}:[\mathrm{M} / \mathrm{H}]=-0.5, \log g \in[4.5,5.0]\right\}$ (dwarf) and $\left\{X^{2}:[\mathrm{M} / \mathrm{H}]=0.0, \log g \in[1.0,2.0]\right\}$ (supergiant). The idea of the method we propose here is the location of all probable solutions $\left\{X^{i}\right\}$ and an estimation of the probability that the star can belong to the solution $\left\{X^{i}\right\}$.

To reduce the number of parameters in the parameter space, we transform the colors of the star into the extinction-free color indices described above. The method is illustrated in the following 5 bands: Johnson $B$ and $V$ magnitudes ( $B, V$ filters are from UBVBUSER code, Buser 1978) and 2MASS $J, H$, and $K_{\mathrm{S}}$ magnitudes (Cutri et al. 2006). Four colors $(B-V, V-J, J-H$, 
$\left.H-K_{\mathrm{S}}\right)$ and three extinction-free color indices $\left(Q_{B V J}, Q_{V J H}\right.$, and $Q_{J H K_{\mathrm{S}}}$ ) are used.

Our method consists of three steps:

1. interval analysis: select all theoretical models in our grid which satisfy the extinction-free color indices of the star;

2. cluster analysis: group all possible solutions in parameter space and find a set of solutions;

3. selection of the best solution: consider all possible solutions to select the only one as the most probable solution for the star in process.

The final solution for the star is a set of parameters (mean values and variances): $\left\{\left\langle T_{\mathrm{eff}}\right\rangle^{i},\langle\log g\rangle^{i},\langle[\mathrm{M} / \mathrm{H}]\rangle^{i},\left\langle A_{V}\right\rangle^{i}, \sigma_{T_{\mathrm{eff}}}^{i}, \sigma_{\log g}^{i}\right.$, $\left.\sigma_{[\mathrm{M} / \mathrm{H}]}^{i}, \sigma_{A_{V}}^{i}\right\}, i \in 1, n$, where $n$ is the number of possible solutions for the star. One of these solutions will be selected as "the most probable" due to criteria we describe below.

\subsection{The first part of the method: interval analysis}

In this step we locate possible solutions in parameter space $\left(\log T_{\text {eff }}, \log g,[\mathrm{M} / \mathrm{H}]\right)$. The table of the theoretical models is constructed with steps $\Delta \log T_{\text {eff }}=0.005 \operatorname{dex}, \Delta \log g=$ $0.1 \mathrm{dex}$, and $\Delta[\mathrm{M} / \mathrm{H}]=0.1 \mathrm{dex}$ and has 249922 entries in the parameter space. For each entry there is one combination of theoretical colors $\left((B-V)^{T},(V-J)^{T},(J-H)^{T},\left(H-K_{\mathrm{S}}\right)^{T}\right)$ and extinction-free color indices $\left(\left(Q_{B V J}\right)_{T},\left(Q_{V J H}\right)_{T},\left(Q_{J H K}\right)_{T}\right)$, as well as the set of supplementary parameters: ratios of extinction $\left(\left(A_{B} / A_{V}\right)^{T},\left(A_{J} / A_{V}\right)^{T},\left(A_{H} / A_{V}\right)^{T},\left(A_{K_{S}} / A_{V}\right)^{T}\right)$, ratios of reddening $\left(\left(E_{B V} / E_{V J}\right)^{T},\left(E_{V J} / E_{J H}\right)^{T},\left(E_{J H} / E_{H K_{\mathrm{S}}}\right)^{T}\right)$. The "observed" values for the star are magnitudes $\left(B^{S}, V^{S}, J^{S}, H^{S}, K_{S}^{S}\right)$ and colors $\left((B-V)^{S},(V-J)^{S},(J-H)^{S},(H-K)_{\mathrm{S}}^{S}\right)$.

\subsubsection{Selection of grid points}

The selection of grid points satisfying the set of "observed" colors is carried out in the space of extinction-free color indices. The value $\left(Q_{B V J}\right)^{T}$ does not depend on extinction. If we know stellar parameters $\left(\log T_{\text {eff }}, \log g,[\mathrm{M} / \mathrm{H}]\right)$, we can find a stellar excess ratio $E_{B V} / E_{V J}^{S}$. Then we can construct an "observed" extinction-free color index $Q_{B V J}^{S}$ and compare it with the theoretical ones $Q_{B V J}^{T}$ to select grid points satisfying to the input index $Q$ :

$Q_{B V J}^{T}=(B-V)^{T}-{\frac{E_{B V}}{E_{V J}}}^{T}(V-J)^{T}=(B-V)^{S}-{\frac{E_{B V}}{E_{V J}}}^{S}(V-J)^{S}$.

The difference between synthetic $Q_{B V J}^{T}=(B-V)^{T}-$ $\left(E_{B V} / E_{V J}\right)^{T}(V-J)^{T}$ and the "observed" index $Q_{B V J}^{S}=(B-V)^{S}-$ $\left(E_{B V} / E_{V J}\right)^{S}(V-J)^{S}$ in this ideal case will be zero. However, we know nothing about parameters of the star except our assumption that the stellar parameters are inside our synthetic grid.

Let us try each of the grid points for the input colors of the star. We take the difference

$\Delta Q_{B V J}=\left|Q_{B V J}^{T}-(B-V)^{S}+{\frac{E_{B V}}{E_{V J}}}^{T}(V-J)^{S}\right|$

in each grid point. We do the same for $\Delta Q_{V J H}$ and $\Delta Q_{J H K_{\mathrm{S}}}$. All points that satisfy the following equations

$$
\begin{aligned}
\Delta Q_{B V J} & <\sigma_{Q_{B V J}} \\
\Delta Q_{V J H} & <\sigma_{Q_{V J H}} \\
\Delta Q_{J H K_{\mathrm{S}}} & <\sigma_{Q_{J H K_{S}}}
\end{aligned}
$$

are selected as possible solutions. The definition of $\sigma_{Q_{B V J}}$ comes from the definition of $Q$ :

$$
\sigma_{Q_{B V J}}^{2}=\sigma_{(B-V)^{S}}^{2}+\left(\frac{E_{B V}{ }^{T}}{E_{V J}}\right)^{2} \sigma_{(V-J)^{S}}^{2}+\left((V-J)^{S}\right)^{2} \sigma_{E_{B V} / E_{V J}{ }^{T}}^{2} .
$$

The problem are the quantities of $\left(E_{B V} / E_{V J}\right)^{T}$ and $\sigma_{E_{B V} / E_{V J}{ }^{T}}^{2}$, which are unknown a priori. In this step we take the maximum value of $\left(E_{B V} / E_{V J}\right)^{T}$ in our theoretical grid (averaged over all points in the grid $\left\langle\left(E_{B V} / E_{V J}\right)^{T}\right\rangle=0.426466 \pm 0.156561$, maximum value $\left.\max \left(E_{B V} / E_{V J}\right)^{T}=0.4516\right)$ for all points, and double the value of $\left(E_{B V} / E_{V J}^{T}\right)^{2} \sigma_{(V-J)^{S}}^{2}$ as a rough estimation of $(V-J)^{S^{2}} \sigma_{E_{B V} / E_{V J}{ }^{T}}^{2}$. Finally,

$\sigma_{Q_{B V J}}^{2}=\sigma_{(B-V)_{S}}^{2}+0.9032 \sigma_{(V-J)_{S}}^{2}$.

The same is done for the estimation of $\sigma_{Q_{V J H}}$ and $\sigma_{Q_{J H K}}$.

After the initial selection of grid points in accordance with Eq. (8) we return from the extinction-free space to the color space and remove all "artifact" entries (those with negative extinction and colors out of the bounding box for the input star).

We assume that the ratio $A_{B} / A_{V}$ is constant for a given temperature, gravity, and metallicity and that it only depends on these parameters and not on $A_{V}$ itself.

For each of the selected grid points, we can find reddening for each color and extinction for each band,

$E_{c}{ }^{S}=c^{S}-c^{T}$

where $c^{S}$ is the observed color for the star and $c^{T}$ is the synthetic color at the selected grid point, $c=(B-V),(V-J),(J-H),(H-$ $\left.K_{\mathrm{S}}\right)$. Also,

$A_{V}^{c}=\left(\frac{A_{V}}{E_{c}}\right)^{T} E_{c}{ }^{S}$

where $\left(A_{V} / E_{c}\right)^{T}$ is the synthetic ratio of extinction in the Johnson $V$ band to color excess in $c$ color at each grid point.

We use the inverse of the variances of the input colors as weights to find the weighted average for the extinction. From the previous equations and the definition of $E_{c}{ }^{S}$ the weights are

$w_{c}=\frac{\left(E_{c} / A_{V}\right)_{T}^{2}}{\sigma_{c}^{2}}$.

To remove negative $A_{V}$, only grid points with $A_{V}>-3 \sigma_{A_{V}}$ were selected, where $\sigma_{A_{V}}$ is a weighted dispersion of $A_{V}$.

For each point in the grid we have theoretical magnitudes, colors, theoretical excess ratios, and an estimation of the extinction $A_{V}$. Now we calculate for each point a synthetic reddened color,

$c_{r}^{T}=c^{T}+\left(\frac{E_{c}}{A_{V}}\right)^{T} A_{V}$

and remove points that are out of the $3 \sigma$ range from the input color of the star. Finally, we select points with

$\left|c_{r}^{T}-c^{S}\right|<3 \sigma_{c^{s}}$

for each color $c$ and have $N$ grid points as possible solutions for the star. 
Table 1. The calibration subsample.

\begin{tabular}{lcccc}
\hline \hline Catalog & Number of & \multicolumn{3}{c}{ Interval in } \\
\cline { 3 - 5 } & stars & $T_{\text {eff }}, \mathrm{K}$ & $\log g$, dex & {$[\mathrm{M} / \mathrm{H}], \mathrm{dex}$} \\
\hline Borkova \& Marsakov (2005) & 876 & $4520-6790$ & $2.94-5.00$ & $-3.85-0.55$ \\
Cayrel de Strobel et al. (1997) & 3247 & $2291-36000$ & $0.09-6.00$ & $-5.6-2.9$ \\
Soubiran et al. (1998) & 211 & $3922-6276$ & $0.58-4.69$ & $-2.91-0.34$ \\
Erspamer \& North (2003) & 140 & $5902-10375$ & $3.04-4.36$ & $-1.08-1.65$ \\
Takeda et al. (2005) & 160 & $5009.3-6967.7$ & $3.185-4.865$ & $-1.291-0.457$ \\
\hline
\end{tabular}

\subsection{The second part of the method: cluster analysis}

We selected $N$ points that satisfy our requirement (Eq. (8)), that are within the allowed extinction range $\left(A_{V}>-3 \sigma_{A_{V}}\right)$, and that are inside the error box of the stellar colors. All these points are possible solutions for the input star must be grouped properly to form a set of possible solutions in the next step.

The distribution of the grid points is uniform in the parameter space $\left(\log T_{\text {eff }}, \log g,[\mathrm{M} / \mathrm{H}]\right)$. We selected all grid points that are inside the error box of stellar colors, so are now able to simulate a more realistic physical distribution of grid points inside the error box assuming a normal distribution of errors of the colors of the star. This step will be made by giving new weights to each selected gridpoint.

The weighted distribution of grid points inside the error box will be projected onto the axes in the parameter space to subdivide the subspace with selected grid points into the set of possible solutions and to assign a probability to each solution.

\subsubsection{Weighting of points}

Suppose that the "observed" star with colors $(B-V)^{S},(V-$ $J)^{S},(J-H)^{S},\left(H-K_{S}\right)^{S}$ has a normal distribution of errors in each color $\left(N\left(0, \sigma_{c}\right)\right)$ and all colors are independent variables. From Eq. (14) we can find the distance of each grid point from the center of the error box (from the observed color of star),

$\Delta c=c_{r}^{T}-c^{S}$,

where $c$ is one of the colors, $c=(B-V),(V-J),(J-H),\left(H-K_{S}\right)$.

The probability that a point occupies the 4-dimensional color space from $(B-V)$ to $(B-V)+\mathrm{d}(B-V),(V-J)$ to $(V-J)+\mathrm{d}(V-J)$, $(J-H)+\mathrm{d}(J-H),\left(H-K_{S}\right)+\mathrm{d}\left(H-K_{\mathrm{S}}\right)$ is

$\mathrm{d} N=\prod_{c=(B-V),(V-J),(J-H),\left(H-K_{S}\right)} f(\Delta c) \mathrm{d} c$,

where

$f(\Delta(B-V))=\mathrm{e}^{-\frac{(\Delta(B-V))^{2}}{\sigma_{B-V}^{2}}}$.

The same distribution for other colors is assumed.

The real distribution of grid points is $N(\Delta(B-V), \Delta(V-$ $J), \Delta(J-H), \Delta(H-K))$. This is a number of points with $\Delta(B-$ $V), \Delta(V-J), \Delta(J-H), \Delta(H-K)$ between $\Delta(B-V)$ and $\Delta(B-$ $V)+\mathrm{d}(B-V), \Delta(V-J)$ and $\Delta(V-J)+\mathrm{d}(V-J)$, and so on; i.e. $N_{p}=N(\Delta(B-V), \Delta(V-J), \Delta(J-H), \Delta(H-K)) \mathrm{d}(B-V) \mathrm{d}(V-$ $J) \mathrm{d}(J-H) d(H-K)$.

The one-dimensional weight for the color $(B-V)$, for example, is $w_{\Delta(B-V)}=f(\Delta(B-V)) \mathrm{d}(B-V)$ and for a point in the 4-dimensional color space

$w^{i}=\frac{\prod_{c=(B-V),(V-J),(J-H),\left(H-K_{S}\right)} w_{\Delta c}}{N_{\mathrm{p}}}$.
The optimal bin in the case of the one-dimensional distribution is $h=3.5 \sigma n^{-1 / 3}$ and, in the case of d dimensions, the optimal bin is $h=O\left(n^{-1 /(d+2)}\right)$ (see Scott et al. 2004). After the weighting of the points we normalise the weight of all selected points to 1 , so that $\sum_{i} w^{i}=1$.

\subsubsection{The final set of solutions}

The distribution of the weighted grid points was projected on each axis in the parameter space $\left(\log T_{\text {eff }}, \log g,[\mathrm{M} / \mathrm{H}]\right)$ to group the grid points into clusters in the parameter space. These clusters are the final set of possible solutions for the input star.

Let us suppose that the grid points are distributed between a minimum $\log T_{\mathrm{eff}}^{\min }$ and a maximum $\log T_{\mathrm{eff}}^{\max }$. We used the original bin of the grid $\left(\Delta \log T_{\text {eff }}=0.005\right.$ dex $)$ to construct the projection of the distribution of $N$ selected grid points on the temperature axis:

$f_{\log T_{\mathrm{eff}}^{i}}=\sum_{j=1}^{N} w_{j}, w_{j}: \log T_{\mathrm{eff}}^{j} \equiv \log T_{\mathrm{eff}}^{i}$,

where $i$ runs through 1 to $M$, and $M$ is the number of bins of the grid between $\log T_{\mathrm{eff}}^{\min }$ and $\log T_{\mathrm{eff}}^{\max }, M=\left(\log T_{\mathrm{eff}}^{\max }-\right.$ $\left.\log T_{\text {eff }}^{\min }\right) / \Delta \log T_{\text {eff. Note that }} \log T_{\text {eff }}, \log g$ and $[\mathrm{M} / \mathrm{H}]$ are discrete values of the grid.

Let us suppose that we have found $L$ local maxima in $\log T_{\text {eff }}$ space. We estimate the significance of each local maximum in accordance with the maximum likelihood criterion for the significance of the maximum (see Materne 1979), i.e. for the $k$ th local maximum the criteria for significance is

$\chi_{k}^{2}=-2 \ln \frac{L_{-k}}{L}$

where

$L=\prod_{j=1}^{M} f_{\log T_{\mathrm{eff}}^{j}}$

and $L_{-k}$ is the likelihood in the absence of the $k$ th local maximum.

We use the $10 \%$ significance level $\left(\chi^{2}>2.7\right)$. If $\chi_{k} \leq 2.7$ the maximum is neglected and joined with the neighboring, most significant maximum. This procedure decreases the number of clusters in $\log T_{\text {eff }}$ space to $N \leq L$. In each of these clusters, we are looking for a clustering on the $\log g$ axis, and for each cluster on the $\log g$ axis, we are looking for the clustering on the metallicity axis with the same procedure. For example, there are $N_{\log T_{\text {eff }}}$ clusters on the temperature axis, the i-th cluster on the temperature axis has $N_{\log g}^{i}$ clusters on the gravity axis and the $j$ th cluster on the gravity axis has $N_{[\mathrm{M} / \mathrm{H}]}^{i, j}$ clusters on the metallicity axis. 
The final number of clusters (and this is the final set of solutions) is

$N_{\text {clusters }}=\sum_{i=1}^{N_{\log T_{\text {eff }}}} \sum_{j=1}^{N_{\log g}^{i}} N_{[\mathrm{M} / \mathrm{H}]}^{i, j}$.

We have distributed $\mathrm{N}$ grid points selected over $N_{\text {clusters }}$ clusters, the sum of weights of points inside the cluster gives us the weight of each cluster:

$W_{j}=\sum w_{i}, w_{i}: i \in N_{j}$,

where $N_{j}$ is the $j$ th cluster.

To find average values of temperature, gravity, and metallicity for each cluster, we repeated the procedure of weighting described in Sect. 4.2.1, this time inside each cluster, and compute the weighted average solutions for $\log T_{\text {eff }}, \log g,[\mathrm{M} / \mathrm{H}]$, $A_{V}$, and distance $r$ with the dispersion of these values inside the cluster.

\subsection{The third part of the method: selection of the solution}

We form a set covering all possible solutions for a star. The final and most complicated step is the selection of one solution from the set.

As we see from Fig. 6 there is no possibility of selecting a single "true" solution in general. Nevertheless we can select "the statistically most probable" solution by the appropriate criteria. We would like to underline that, by these criteria, we do not use guesses about the astrophysical parameters themselves, so the selection at this point does not introduce limitations on the parameters.

There are a number of methods for this selection: we can take the solution with the largest weight or with minimal distance from the input "observed" color in color space or take one with an unbiased distribution of grid points around the center of the error box in color space.

Let us show an example of the determination of parameters to point out the major troubles we have to face. We used the point from the synthetic grid with $T_{\text {eff }}=4000 \mathrm{~K}, \log g=4.45$, and $[\mathrm{M} / \mathrm{H}]=0.0$, photometric errors equal to $0.05 \mathrm{mag}$ in all colors were taken. The first step of the method returned 6000 points distributed between $3664 \mathrm{~K}$ and $4943 \mathrm{~K}$ in temperature, between 0.3 and 5.0 dex in $\log g$ and between -2.5 and 0.5 dex in metallicity. All points were weighted and grouped in 5 possible solutions (see Table 2). The second part of the method (the cluster analysis) is illustrated by Fig. 7. The first maximum on the $\log T_{\text {eff }}$ axis is rejected by the significance level assumed. Finally Fig. 8 presents the clusters in the projections onto the $\log T_{\text {eff }}-$ $\log g$ and the $\log T_{\text {eff }}-[\mathrm{M} / \mathrm{H}]$ planes.

We define the following two quantities that describe the error of the estimated parameter:

1. the offset (bias) of the selected solution from the true parameters $\Delta \log T_{\text {eff }}, \Delta \log g, \Delta[\mathrm{M} / \mathrm{H}]$. These values are the differences between the estimated parameter (the center of the selected cluster) and the true parameter known only in simulations;

2. the dispersion of the estimated parameter $\sigma_{\log T_{\text {eff }}}, \sigma_{\log g}$, $\sigma_{[\mathrm{M} / \mathrm{H}]}$.

From Fig. 8 we see that it is possible to subdivide the first cluster (first solution in Table 2). This possibility was ruled out by the significance level we assumed (see Fig. 7, the first peak on the

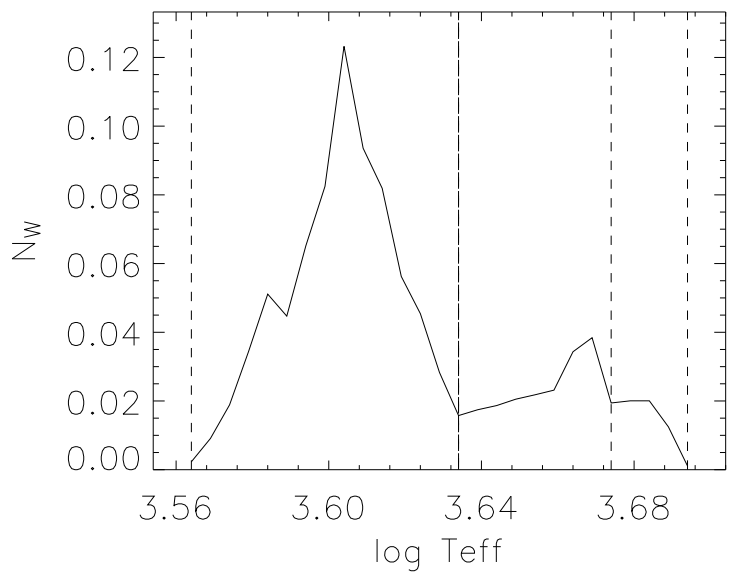

Fig. 7. The projection of the weighted distribution function of selected grid points on the $\log T_{\text {eff }}$ axis, showing the subdivision made with an assumed $10 \%$ significance level.
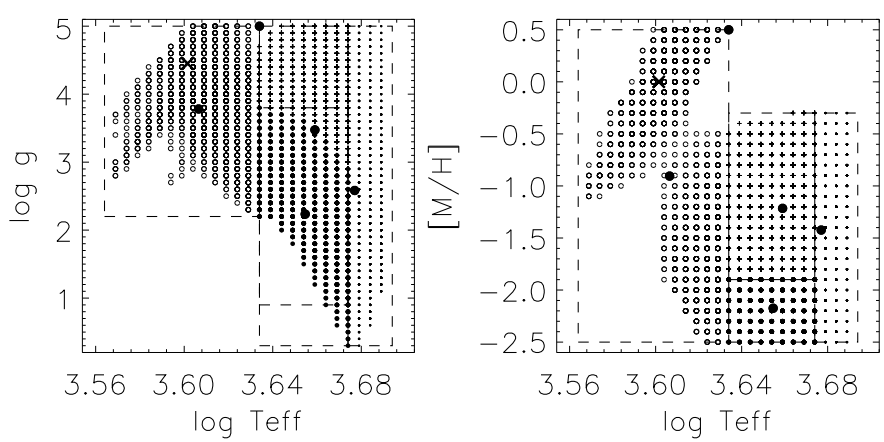

Fig. 8. Points selected for the test input point in the parameter space. Big open circles are for solution 1 (see Table 2), big closed circles for solution 2, crosses for solution 3 , and small circles are for solution 5 .

distribution function). If we decrease the significance level, we are increasing the number of clusters in the set of solutions but decrease the weight for each cluster. Selecting a single cluster we face the problem of choosing between a number of clusters: the one that includes the true parameters of a star we call "true" cluster (solution) for the star, the remaining are "false" solutions. In practice we can distinguish between "true" and "false" solutions only if we knew the parameters of the star a priori; however, we can determine the region in the parameter space where only a single, hence "true", solution is available or where the "true" solution can be selected from a set of solutions by some criteria. We note that the definition of this region in parameter space depends on the desired precision of the determination of parameters. We show below how to define such a "most preferable" region.

\subsubsection{The most preferable regions in parameter space}

We have determined regions in the parameter space where we can best estimate astrophysical parameters; in other words, if the solution falls into this region, we can be sure that this estimation is a correct, unbiased solution for the star.

To find the most preferable regions, we performed simulations using the synthetic grid points as input "observed" colors. We interpolated between the grid points to create from a grid point with $\left\{\log T_{\text {eff }}, \log g,[\mathrm{M} / \mathrm{H}]\right\}$ new points with $\left\{\log T_{\text {eff }}+\right.$ $\left.i \Delta \log T_{\text {eff }} / 2, \log g+j \Delta \log g / 2,[\mathrm{M} / \mathrm{H}]+k \Delta[\mathrm{M} / \mathrm{H}] / 2\right\}, i, j, k=$ $[0,1]$, and $\Delta \log T_{\mathrm{eff}}, \Delta \log g$, and $\Delta[\mathrm{M} / \mathrm{H}]$ are intervals between points of the initial grid in temperature, gravity, and metallicity. 
Table 2. Set of solutions for a test point of $T_{\text {eff }}=4000 \mathrm{~K}, \log g=4.45$ and $[\mathrm{M} / \mathrm{H}]=0.0$ and with an assumed error in color of $0.05 \mathrm{mag}$.

\begin{tabular}{ccccccccccc}
\hline \hline $\begin{array}{c}\text { Number } \\
\text { of points }\end{array}$ & $\begin{array}{c}\text { Total } \\
\text { weight }\end{array}$ & $\begin{array}{c}T_{\text {eff }}^{\min }, \\
\mathrm{K}\end{array}$ & $\begin{array}{c}T_{\text {eff }}^{\max }, \\
\mathrm{K}\end{array}$ & $\begin{array}{c}T_{\text {eff }}^{w} \pm \sigma_{T_{\text {eff }}}, \\
\mathrm{K}\end{array}$ & $\log g^{\min }$, & $\log g^{\max }$, & $\log g^{w} \pm \sigma_{\log g}$, & {$[\mathrm{M} / \mathrm{H}]^{\min }$} & {$[\mathrm{M} / \mathrm{H}]^{\mathrm{max}}$} & {$[\mathrm{M} / \mathrm{H}]^{w} \pm \sigma_{[\mathrm{M} / \mathrm{H}]}$} \\
\hline 1713 & 0.7204 & 3664 & 4305 & $4041_{+153}^{-147}$ & 2.2 & 5.0 & $3.8 \pm 0.4$ & -2.5 & 0.5 & $-0.9 \pm 0.8$ \\
951 & 0.0744 & 4305 & 4721 & $4515_{+156}^{-151}$ & 0.3 & 3.8 & $2.2 \pm 0.5$ & -2.5 & -1.9 & $-2.2 \pm 0.04$ \\
2736 & 0.1346 & 4305 & 4721 & $4561_{+145}^{-145}$ & 0.9 & 5.0 & $3.5 \pm 0.7$ & -1.9 & -0.3 & $-1.2 \pm 0.2$ \\
1 & 0.0006 & 4305 & 4305 & 4305 & 5.0 & 5.0 & 5.0 & 0.5 & 0.5 & 0.5 \\
1728 & 0.0700 & 4721 & 4943 & $4754_{+51}^{-51}$ & 0.3 & 5.0 & $2.6 \pm 1.3$ & -2.5 & -0.3 & $-1.4 \pm 0.4$ \\
\hline
\end{tabular}

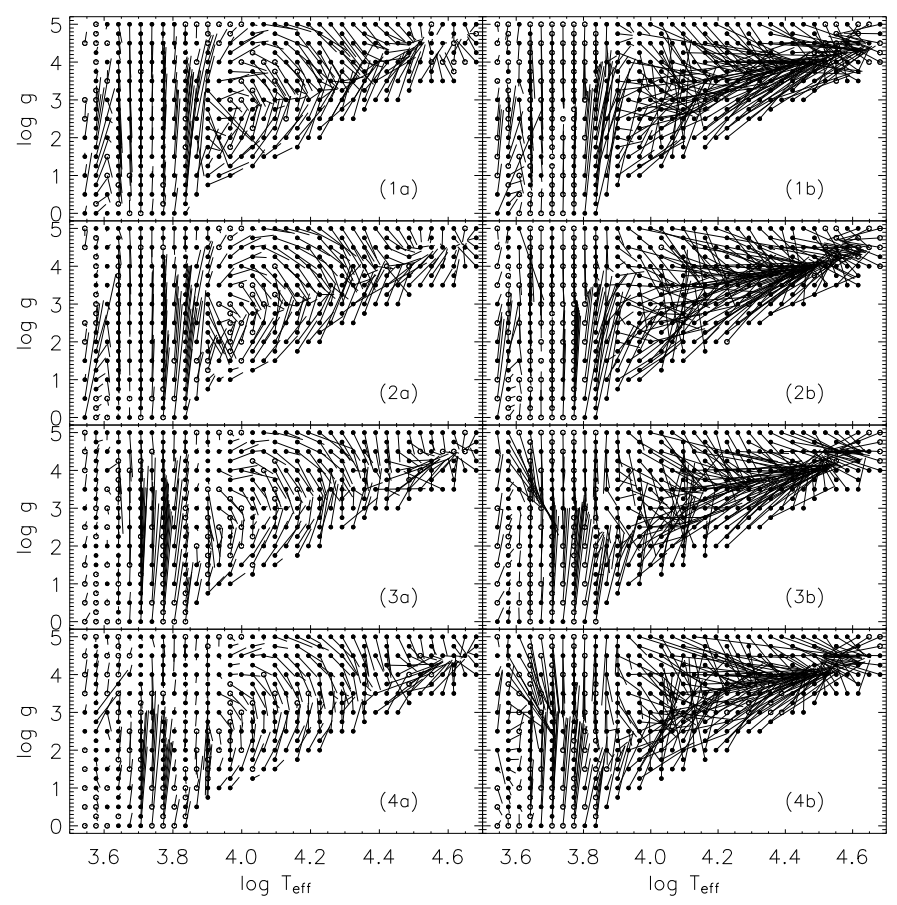

Fig. 9. The offset (bias) in the case of the selection of the solution with the largest weight in the $\left(\log T_{\text {eff }}, \log g\right)$ plane. Open circles are the cases of a single solution, lines show the distance between the selected solution and the input grid point. Panel 1a) is for metallicity $[\mathrm{M} / \mathrm{H}]=-2.0$ dex and an assumed rms-error $\sigma_{\mathrm{ph}}=0.01 \mathrm{mag}$ in all colors, panel 1a) for metallicity $[\mathrm{M} / \mathrm{H}]=-2.0 \mathrm{dex}$ and an assumed rmserror $\sigma_{\mathrm{ph}}=0.05 \mathrm{mag}$ in all colors, panel 2a) $-[\mathrm{M} / \mathrm{H}]=-1.0 \mathrm{dex}$ and $\sigma_{\mathrm{ph}}=0.01 \mathrm{mag}$, panel 2b) $-[\mathrm{M} / \mathrm{H}]=-1.0 \mathrm{dex}$ and $\sigma_{\mathrm{ph}}=0.05 \mathrm{mag}$, panel 3a) $-[\mathrm{M} / \mathrm{H}]=0.0$ dex and $\sigma_{\mathrm{ph}}=0.01 \mathrm{mag}$, panel 3b) $[\mathrm{M} / \mathrm{H}]=0.0$ dex and $\sigma_{\mathrm{ph}}=0.05 \mathrm{mag}$, panel 4a) $-[\mathrm{M} / \mathrm{H}]=0.3 \mathrm{dex}$ and $\sigma_{\mathrm{ph}}=0.01 \mathrm{mag}$, panel $\left.\mathbf{4 b}\right)-[\mathrm{M} / \mathrm{H}]=0.3 \mathrm{dex}$ and $\sigma_{\mathrm{ph}}=0.05 \mathrm{mag}$.

For each point of this enhanced grid, we calculated synthetic colors. The resulting test grid includes points with the largest scatter from the points of the initial grid in the parameter space.

We tested the method on two sets of errors in colors; in the first case, all colors had an error $0.01 \mathrm{mag}$ and in the second all colors had an error $0.05 \mathrm{mag}$. Figure 9 shows the solution selected by the maximum weight with offsets (biases) caused by the selection of the "false" cluster or the shift inside the selected "true" cluster, and Fig. 11 is the same for the temperaturemetallicity plane. Figure 10 plots the rms-errors of the selected solution for the temperature - gravity plane and Fig. 12 - for the temperature-metallicity plane.

We define a region in the parameter space as suitable for the unbiased determination of astrophysical parameters, if we can select a solution for the input point inside the region, and this final solution has biases for temperature $\Delta \log T_{\text {eff }}<0.05$, for gravity $\Delta \log g<0.3$, rms-errors $\sigma_{\log T_{\text {eff }}}<0.05, \sigma_{\log g}<0.3$ in

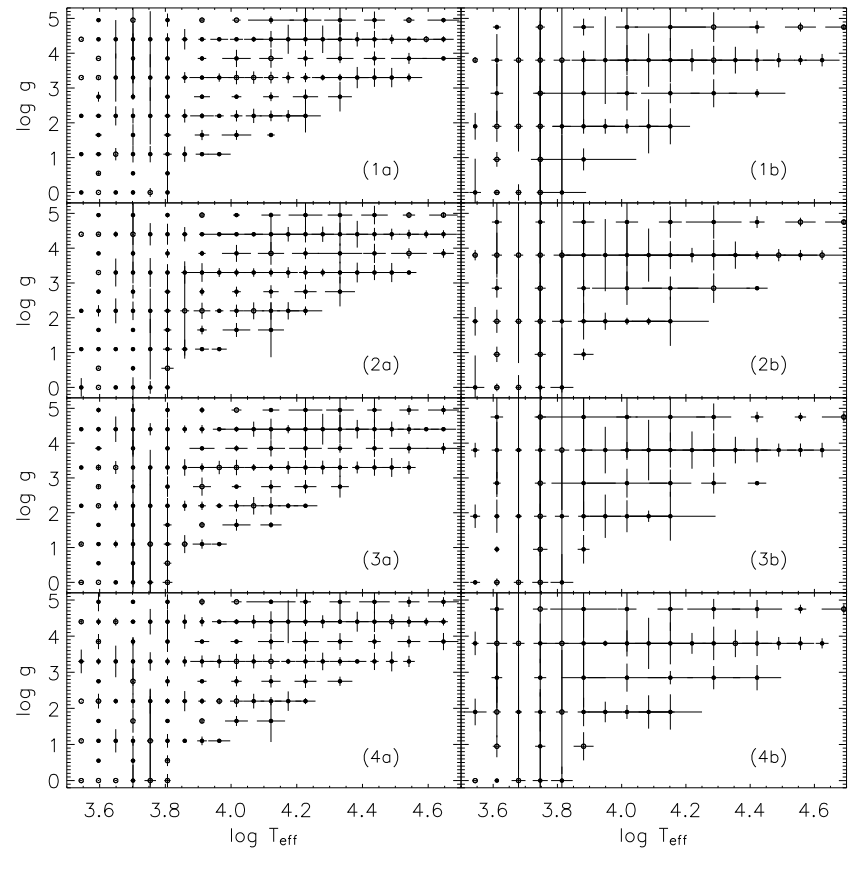

Fig. 10. Errors of the selected solution on the $\left(\log T_{\text {eff }}, \log g\right)$ plane. Panel 1a) is for metallicity $[\mathrm{M} / \mathrm{H}]=-2.0 \mathrm{dex}$ and an assumed error $\sigma_{\mathrm{ph}}=0.01 \mathrm{mag}$ in all colors, panel 1a) for metallicity $[\mathrm{M} / \mathrm{H}]=$ $-2.0 \mathrm{dex}$ and an assumed error $\sigma_{\mathrm{ph}}=0.05 \mathrm{mag}$ in all colors, panel 2a) $[\mathrm{M} / \mathrm{H}]=-1.0 \mathrm{dex}$ and $\sigma_{\mathrm{ph}}=0.01 \mathrm{mag}$, panel $\left.2 \mathbf{b}\right)-[\mathrm{M} / \mathrm{H}]=-1.0 \mathrm{dex}$ and $\sigma_{\mathrm{ph}}=0.05 \mathrm{mag}$, panel 3a) $-[\mathrm{M} / \mathrm{H}]=0.0 \mathrm{dex}$ and $\sigma_{\mathrm{ph}}=0.01 \mathrm{mag}$, panel 3b) $-[\mathrm{M} / \mathrm{H}]=0.0$ dex and $\sigma_{\mathrm{ph}}=0.05 \mathrm{mag}$, panel 4a) $[\mathrm{M} / \mathrm{H}]=0.3$ dex and $\sigma_{\mathrm{ph}}=0.01 \mathrm{mag}$, panel $\left.4 \mathbf{b}\right)-[\mathrm{M} / \mathrm{H}]=0.3 \operatorname{dex}$ and $\sigma_{\mathrm{ph}}=0.05 \mathrm{mag}$.

the temperature-gravity plane and $\Delta \log T_{\text {eff }}<0.05, \Delta[\mathrm{M} / \mathrm{H}]<$ 0.3 , rms-errors $\sigma_{\log T_{\text {eff }}}<0.05, \sigma_{[\mathrm{M} / \mathrm{H}]}<0.3$ in the temperaturemetallicity plane. This is done in Figs. 13 and 14. According to these figures we define the following regions in the $\log T_{\text {eff }}-$ $\log g$ plane

$\log T_{\text {eff }}<3.75, \log g>4.0$;

$\log T_{\text {eff }} \in[3.395,3.7], \log g<1.0$;

$\log T_{\text {eff }} \in[3.8,4.05], \log g>4.5$;

$\log T_{\text {eff }} \in[3.9,4.2], \log g \in[2,3]$;

$\log T_{\text {eff }}>4.4, \log g>4.0$

as the most suitable for the determination of $\log T_{\text {eff }}$ and $\log g$, and on the $\log T_{\text {eff }}-[\mathrm{M} / \mathrm{H}]$ plane

$\log T_{\text {eff }} \in[3.7,3.8]$

as the most suitable in the search for metallicity. We note that the definition of these regions depends on the assumed errors of photometry, i.e. observations; and in the case of real stars with 


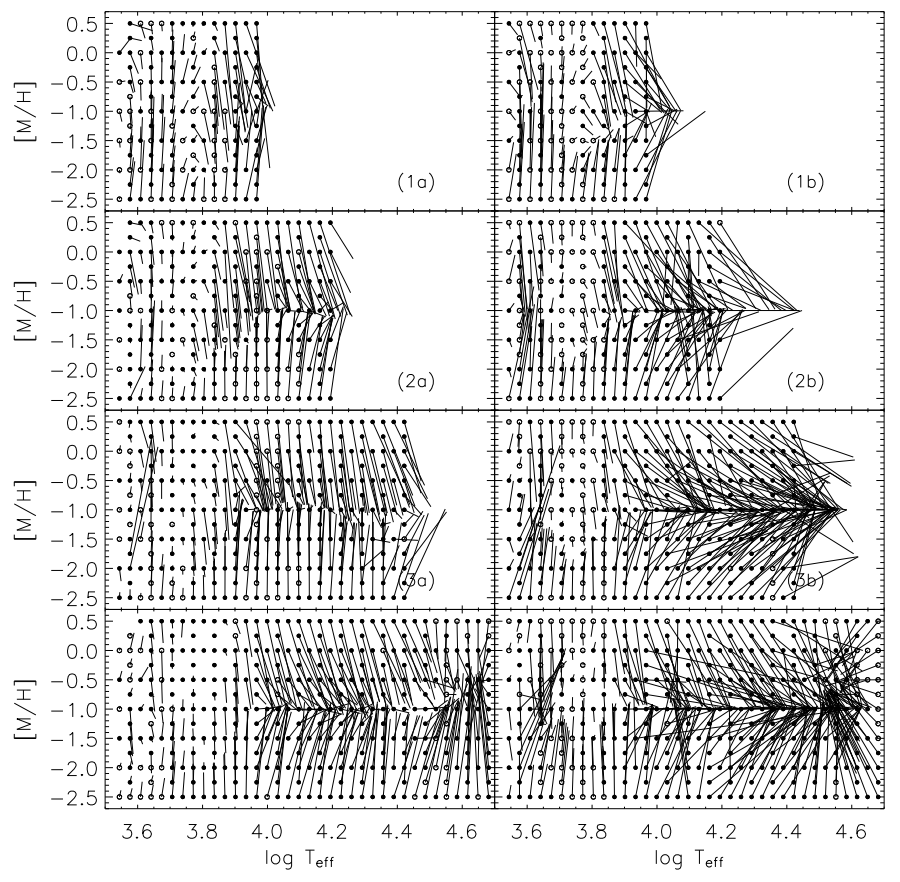

Fig. 11. The bias in the case of the selection of the solution with the largest weight in the $\left(\log T_{\text {eff }},[\mathrm{M} / \mathrm{H}]\right)$ plane. Open circles are the cases of a single solution, lines show the distance between the selected solution and the input grid point. Panel 1a) is for $\log g=1.0$ dex and an assumed error $\sigma_{\mathrm{ph}}=0.01 \mathrm{mag}$ in all colors, panel 1a) for $\log g=1.0 \mathrm{dex}$ and an assumed error $\sigma_{\mathrm{ph}}=0.05 \mathrm{mag}$ in all colors, panel 2a) $\log g=2.0$ dex and $\sigma_{\text {ph }}=0.01$ mag, panel 2b) $-\log g=2.0$ dex and $\sigma_{\mathrm{ph}}=0.05 \mathrm{mag}$, panel 3a) $-\log g=3.0$ dex and $\sigma_{\mathrm{ph}}=0.01 \mathrm{mag}$, panel 3b) $-\log g=3.0 \mathrm{dex}$ and $\sigma_{\mathrm{ph}}=0.05 \mathrm{mag}$, panel 4a) $\log g=4.5 \mathrm{dex}$ and $\sigma_{\mathrm{ph}}=0.01 \mathrm{mag}$, panel 4b) $-\log g=4.5 \mathrm{dex}$ and $\sigma_{\mathrm{ph}}=0.05 \mathrm{mag}$.

different observational errors in different bands, the definition of the most suitable regions can vary.

\subsubsection{Setting bounds on extinction}

Estimating the most preferable regions for our method made in the previous section is based on the assumption that photometric errors are the same in all bands. In the case of real observations, we would have to recalculate the estimation of errors and biases at any point on the synthetic grid for each combination of errors of colors for the input stars. This is possible but consumes enormous computational resources and slows the estimation.

For the actual application of the method to observations, we propose the following procedure: we estimate the extinction per photometric distance. We assume a maximum extinction in the galactic plane to be $2.0 \mathrm{mag} / \mathrm{kpc}$ in the Johnson $V$ band and a minimum extinction in the direction of the galactic poles as $0.1 \mathrm{mag} / \mathrm{kpc}$ (from Schlegel et al. 1998).

Finally there are three criteria for selecting a single solution for the star:

1. selection of the solution with the largest weight;

2 . selection based on the most preferable region in the parameter space;

3. selection based on setting a bound on the extinction per photometric distance.

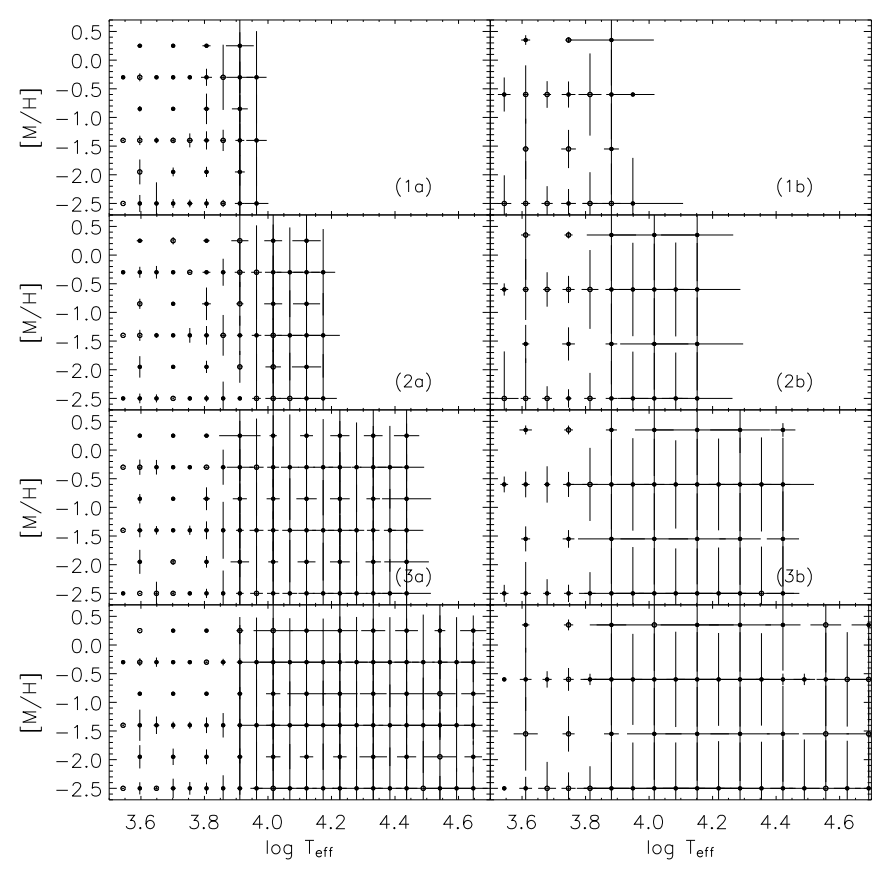

Fig. 12. Errors in the case of the selection of the solution with the biggest weight on the $\left(\log T_{\text {eff }},[\mathrm{M} / \mathrm{H}]\right)$ plane. Panel 1a) is for $\log g=$ $1.0 \mathrm{dex}$ and an assumed error $\sigma_{\mathrm{ph}}=0.01 \mathrm{mag}$ in all colors, panel 1a) is for $\log g=1.0 \mathrm{dex}$ and an assumed error $\sigma_{\mathrm{ph}}=0.05 \mathrm{mag}$ in all colors, panel 2a) $-\log g=2.0$ dex and $\sigma_{\mathrm{ph}}=0.01 \mathrm{mag}$, panel 2b) $\log g=2.0$ dex and $\sigma_{\mathrm{ph}}=0.05 \mathrm{mag}$, panel 3a) $-\log g=3.0 \mathrm{dex}$ and $\sigma_{\mathrm{ph}}=0.01 \mathrm{mag}$, panel 3b) $-\log g=3.0 \mathrm{dex}$ and $\sigma_{\mathrm{ph}}=0.05 \mathrm{mag}$, panel 4a) $-\log g=4.5 \mathrm{dex}$ and $\sigma_{\mathrm{ph}}=0.01 \mathrm{mag}$, panel 4b) $\log g=4.5$ dex and $\sigma_{\mathrm{ph}}=0.05 \mathrm{mag}$.

\section{The method used in practice and problems of the method}

We used the 2096 stars of our compiled catalog (see Sect. 3 for details) with Johnson $B, V$, and 2 MASS $J, H, K_{\mathrm{S}}$ photometry to test the method in practice.

Table 3 shows the statistics for the different combinations of the criteria described in the previous section: criterion 1 , criterion 1 combined with criterion 2 for selected stars (we select a single solution with the largest weight for each star and select stars within the most preferable regions), and criterion 1 combined with criterion 3 for selected stars. The last 3 columns in the table show the means and variances of the differences between the "true" spectroscopic parameter and our estimation (for

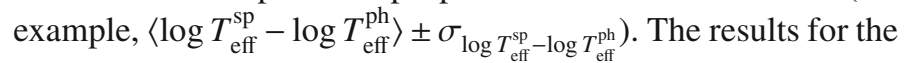
calibration subset, based on the different selection criteria are shown in Figs. 15-17 (left panel for criterion 1, middle panel for criterion $1 \& 2$, right panel for criterion $1 \& 3$ ).

The results for all stars in the compiled catalogue are shown in Figs. 18-20. We selected stars that have solutions with weight $w_{n}>0.8$, uncertainties of the input photometry better that $0.05 \mathrm{mag}, \mathrm{rms}-\mathrm{errors}$ of the estimated parameters (dispersion of the points around the weighted average parameter for the solution) better that $0.05 \mathrm{dex}$ for $\log T_{\text {eff }}, 0.5 \mathrm{dex}$ for $\log g$, and 0.3 dex for $[\mathrm{M} / \mathrm{H}]$. It becomes clear that gravity is the least accurate unknown in estimating of astrophysical parameters: in the case of calibration catalog we were able to estimate gravity only for $2 \%$ of the stars with an appropriate precision (better than $0.5 \mathrm{dex})$. We note that only 1186 stars from the calibration catalog have photometric errors better than 0.05 mag and 
Table 3. Selection of the solution: comparison of different cases.

\begin{tabular}{|c|c|c|c|c|c|c|}
\hline \multirow{2}{*}{ Parameter } & \multicolumn{3}{|c|}{ Number of stars } & \multicolumn{3}{|c|}{ average and variance } \\
\hline & $\begin{array}{c}\text { criterion } \\
1\end{array}$ & $\begin{array}{l}\text { criterion } \\
1 \& 2\end{array}$ & $\begin{array}{c}\text { criterion } \\
1 \& 3\end{array}$ & $\begin{array}{c}\text { criterion } \\
1\end{array}$ & $\begin{array}{l}\text { criterion } \\
1 \& 2\end{array}$ & $\begin{array}{c}\text { criterion } \\
1 \& 3\end{array}$ \\
\hline $\log T_{\mathrm{eff}}^{\mathrm{sp}}-\log T_{\mathrm{eff}}^{\mathrm{ph}}$ & 588 & 31 & 330 & $0.001 \pm 0.059$ & $0.054 \pm 0.176$ & $-0.010 \pm 0.025$ \\
\hline $\log g^{\mathrm{sp}}-\log g^{\mathrm{ph}}$ & 133 & 38 & 40 & $-0.166 \pm 0.924$ & $-0.336 \pm 1.047$ & $0.191 \pm 0.778$ \\
\hline$[\mathrm{M} / \mathrm{H}]^{\mathrm{sp}}-[\mathrm{M} / \mathrm{H}]^{\mathrm{ph}}$ & 527 & 199 & 305 & $0.011 \pm 0.457$ & $-0.029 \pm 0.459$ & $0.017 \pm 0.407$ \\
\hline
\end{tabular}

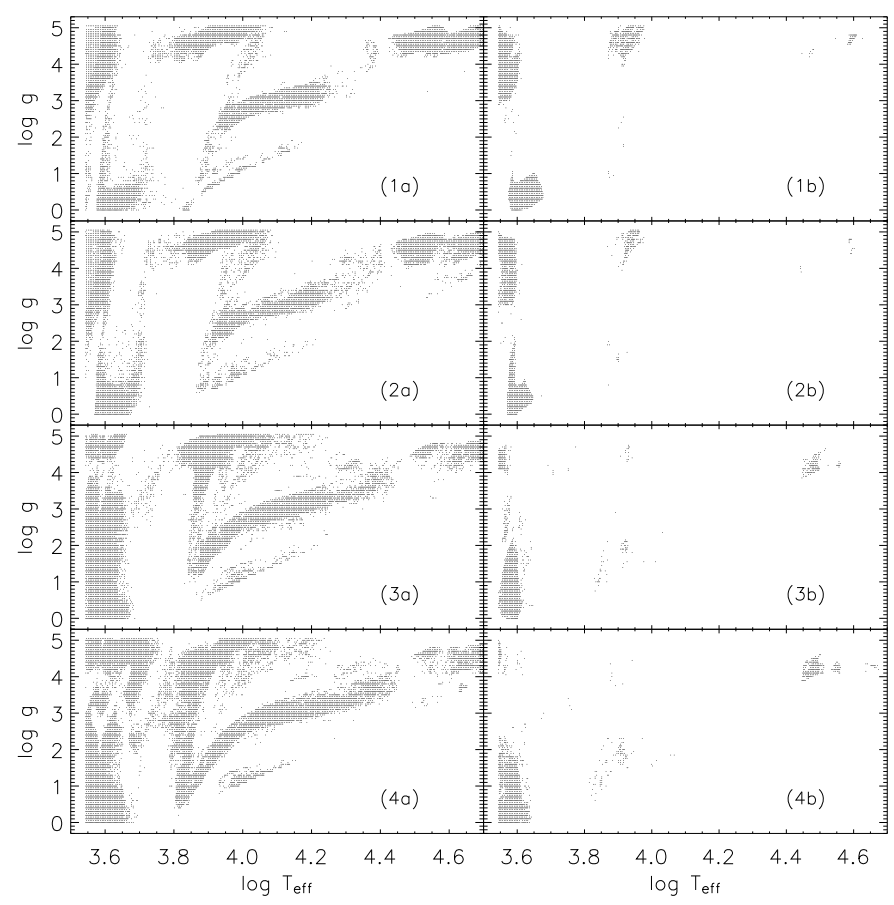

Fig. 13. The best regions for estimating astrophysical parameters on the $\left(\log T_{\text {eff }}, \log g\right)$ plane. Biases and errors of the solution (see text for the explanation) are in the range: $\Delta \log T_{\text {eff }}<0.05, \sigma_{\log T_{\text {eff }}}<0.05$, $\Delta \log g<0.3, \sigma_{\log g}<0.3$. Panel 1a) is for metallicity $[\mathrm{M} / \mathrm{H}]=$ $-2.0 \mathrm{dex}$ and an assumed error $\sigma_{\mathrm{ph}}=0.01 \mathrm{mag}$ in all colors, panel 1a) for metallicity $[\mathrm{M} / \mathrm{H}]=-2.0$ dex and an assumed error $\sigma_{\mathrm{ph}}=0.05 \mathrm{mag}$ in all colors, panel 2a) $-[\mathrm{M} / \mathrm{H}]=-1.0$ dex and $\sigma_{\mathrm{ph}}=0.01 \mathrm{mag}$, panel 2b) $-[\mathrm{M} / \mathrm{H}]=-1.0$ dex and $\sigma_{\mathrm{ph}}=0.05 \mathrm{mag}$, panel 3a) $[\mathrm{M} / \mathrm{H}]=0.0$ dex and $\sigma_{\mathrm{ph}}=0.01 \mathrm{mag}$, panel 3b) $-[\mathrm{M} / \mathrm{H}]=0.0 \mathrm{dex}$ and $\sigma_{\mathrm{ph}}=0.05 \mathrm{mag}$, panel $\left.\mathbf{4 a}\right)-[\mathrm{M} / \mathrm{H}]=0.3 \mathrm{dex}$ and $\sigma_{\mathrm{ph}}=0.01 \mathrm{mag}$, panel 4b) $-[\mathrm{M} / \mathrm{H}]=0.3$ dex and $\sigma_{\mathrm{ph}}=0.05 \mathrm{mag}$.

only 21 stars have photometric errors better than 0.02 mag in all bands. We discuss the reasons for failures in the estimation of parameters below. We conclude that the combination of criteria $1 \& 3$ works best in selecting stars with the best estimation of astrophysical parameters.

\subsection{The problem of the selection of the synthetic model grid}

There are a number of libraries of synthetic models of atmospheres is available today. The most popular among them are the BaSeL library based on Kurucz models with an addition of synthetic and semi-empirical models for cool stars (Lejeune et al. 1997), original Kurucz models (ATLAS9 program, Castelli \& Kurucz 2003), and MARCS models (Gustafsson et al. 2003). Even the models of the same author for the same parameters (microturbulent velocity etc.) differ due to improvements that the authors introduced into different versions of models.

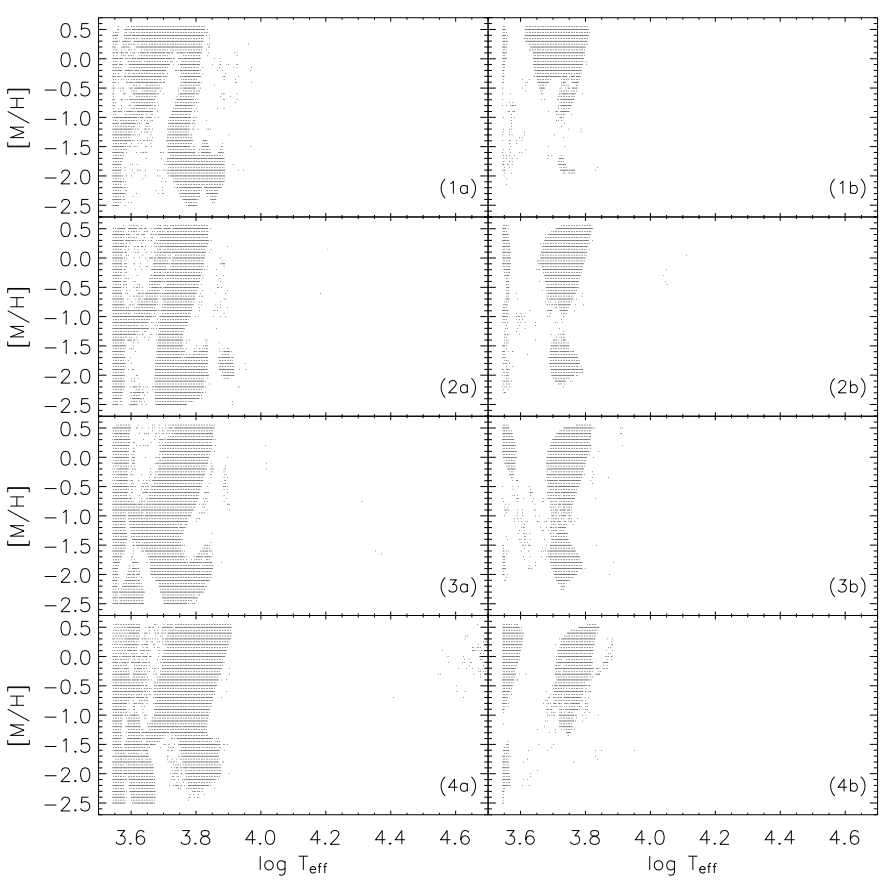

Fig. 14. The best regions for estimating astrophysical parameters on the $\left(\log T_{\text {eff }},[\mathrm{M} / \mathrm{H}]\right)$ plane. Biases and errors of the solution (see text for the explanation) are in the range: $\Delta \log T_{\text {eff }}<0.05, \sigma_{\log T_{\text {eff }}}<0.05$, $\Delta[\mathrm{M} / \mathrm{H}]<0.3, \sigma_{[\mathrm{M} / \mathrm{H}]}<0.3$. Panel 1a) is for $\log g=1.0 \mathrm{dex}$ and an assumed error $\sigma_{\mathrm{ph}}=0.01 \mathrm{mag}$ in all colors, panel 1a) for $\log g=$ $1.0 \mathrm{dex}$ and an assumed error $\sigma_{\mathrm{ph}}=0.05 \mathrm{mag}$ in all colors, panel 2a) $-\log g=2.0$ dex and $\sigma_{\mathrm{ph}}=0.01 \mathrm{mag}$, panel 2b) $-\log g=2.0 \mathrm{dex}$ and $\sigma_{\mathrm{ph}}=0.05 \mathrm{mag}$, panel 3a) $-\log g=3.0 \mathrm{dex}$ and $\sigma_{\mathrm{ph}}=0.01 \mathrm{mag}$, panel 3b) $-\log g=3.0$ dex and $\sigma_{\mathrm{ph}}=0.05 \mathrm{mag}$, panel 4a) $-\log g=$ $4.5 \mathrm{dex}$ and $\sigma_{\mathrm{ph}}=0.01 \mathrm{mag}$, panel $\left.4 \mathbf{b}\right)-\log g=4.5 \mathrm{dex}$ and $\sigma_{\mathrm{ph}}=$ 0.05 mag.

We do not discuss the different choices of theoretical models leaving this subject to the user of the method, but we note that some of the discrepancies in the parameters estimated via our method in comparison with the parameters from spectroscopic estimations can be due to the different model sets used for photometric (performed by us) and spectroscopic (represented in the calibration catalog) estimations of astrophysical parameters.

The direct comparison of Kurucz models with the SEDs of real stars (see, for example, Straižys et al. 1997) shows problems with the ultraviolet part of spectra for main-sequence stars, as well as an inconsistency in the synthetic SEDs with real SEDs for late type stars (K7-M stars). This will not influence our results for real stars as the lowest temperature estimation in Fig. 18 turned out to be $4200 \mathrm{~K}$ but must be taken into consideration in future work as late type stars are in the area with the best estimation of parameters possible (see Figs. 13 and 14).

Both photometric and spectroscopic determinations of astrophysical parameters based on synthetic models suffer from the uncertainties and systematic errors of the selected synthetic grid. 


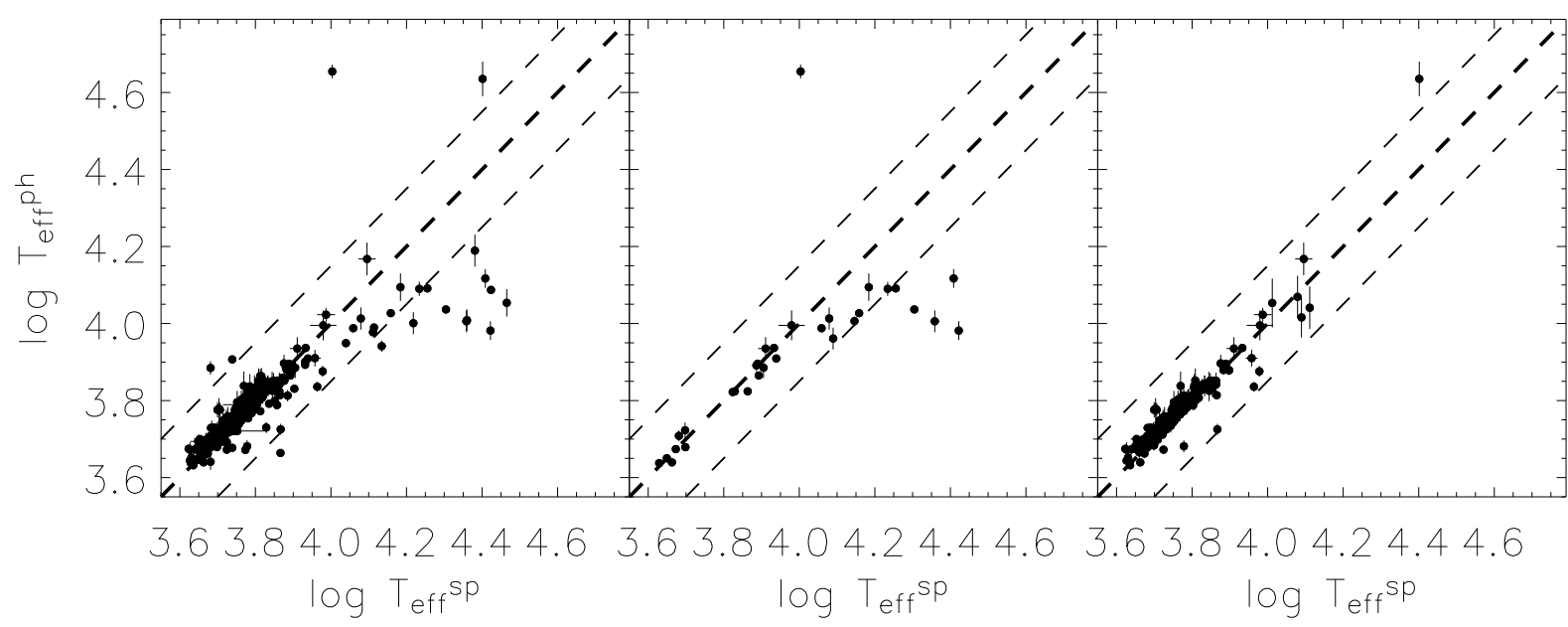

Fig. 15. The test of the method on the calibration sample estimating temperature. In the left panel there are all stars with $\sigma_{\text {log }} T_{\text {eff }}<0.05$ for an estimated temperature and errors of photometry better than 0.05 mag in all colors. The middle panel shows a subsample of the left panel falling into the most suitable region on the $\left(\log T_{\text {eff }}, \log g\right)$ plane as described in the text and the right panel gives a subsample of the left panel with $A_{V}[\mathrm{mag}] / d[\mathrm{kpc}] \in[0.1,2.0]$. The dashed lines confine the $3-\sigma$ region; i.e, they are the median $\operatorname{line} \operatorname{and} \log T_{\mathrm{eff}}^{\mathrm{sp}}=\log T_{\mathrm{eff}}^{\mathrm{ph}}+0.15, \log T_{\mathrm{eff}}^{\mathrm{sp}}=$ $\log T_{\mathrm{eff}}^{\mathrm{ph}}-0.15$ lines.

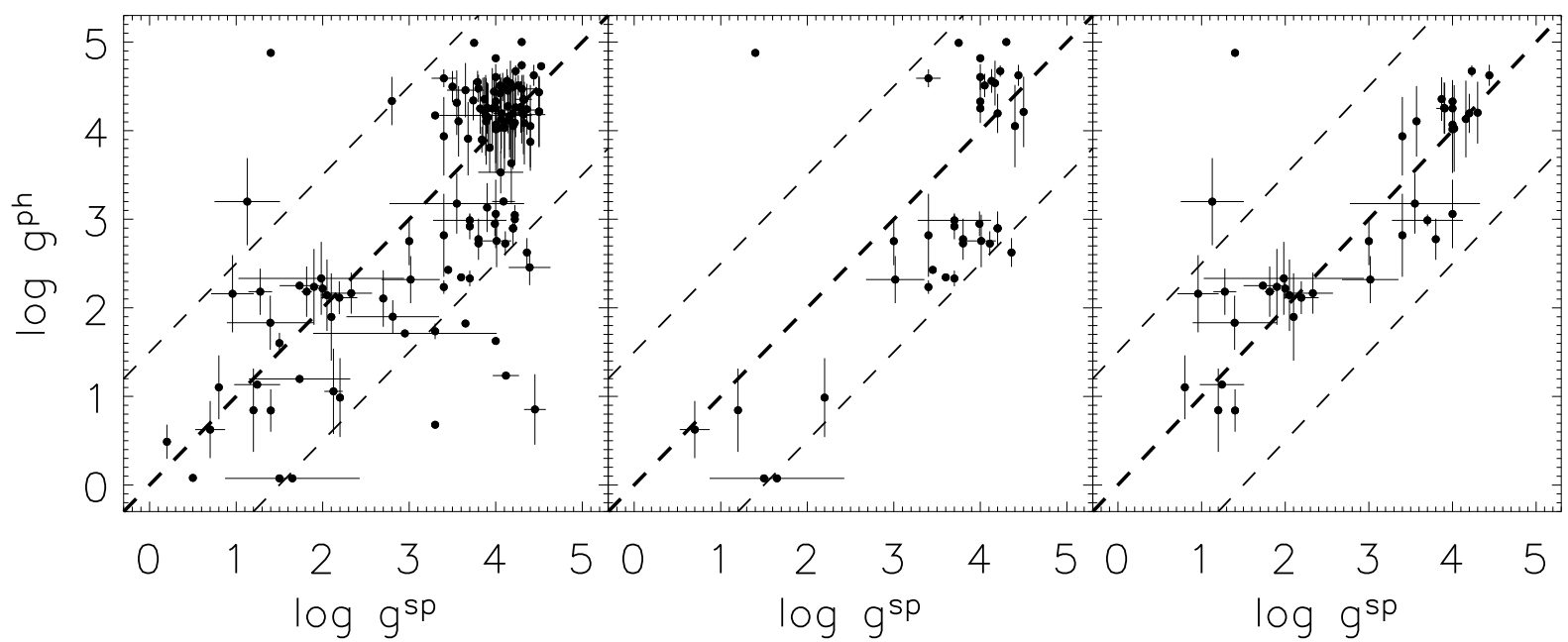

Fig. 16. The test of the method on the calibration sample for estimating gravity. In the left panel there are all stars with $\sigma_{\log g}<0.5$ for the estimated gravity and photometric errors better than 0.05 mag in all colors. The middle panel shows a subsample of the left panel falling into the most suitable region on the $\left(\log T_{\text {eff }}, \log g\right)$ plane as described in the text, and in the right panel, we plot a subsample of the left panel with $A_{V}[\mathrm{mag}] / d[\mathrm{kpc}] \in[0.1,2.0]$. The dashed lines are the median line and $\log g^{\mathrm{sp}}=\log g^{\mathrm{ph}}+1.5, \log g^{\mathrm{sp}}=\log g^{\mathrm{ph}}-1.5 \operatorname{lines}$.

We urge the users of this method to always make a reference to the synthetic models used and to accept the resulting parameters as parameters in the system of the selected synthetic models. A detailed study of the systematic errors of astrophysical parameters due to problems of synthetic models is beyond the scope of this paper. There are many reasons for such systematics, for example, the convection treatment adopted for models and the influence of models on fundamental parameters is discussed in Heiter et al. (2002), who estimate that the systematic effect in temperature could be up to $400 \mathrm{~K}$.

The estimation of the systematics in the photometry due to the difference between synthetic models (Martins \& Coelho 2007) shows that, for Johnson $B, V, J, H$, and $K$ bands, they are within $0.05 \mathrm{mag}$, which can be accepted as an upper limit for this type of uncertainties in photometry. The rough estimation of the influence of these uncertainties on the resulting parameters can be done in the same way as the estimation of biases due to errors in the input photometry (Figs. 13 and 14).

\subsection{The problem of stars outside the grid}

As we can see from the previous section and Figs. 15-17 we reached quite good agreement between spectroscopic and photometric estimation of astrophysical parameters, however there are some cases of a large differences between our estimated parameters and parameters from spectroscopy. To understand the reason for these differences we searched the Simbad database for the information about each star with "true" (spectroscopic) parameter out of the 3- $\sigma$ range from the estimated parameters. Table 4 summarizes the results and indicates the reason for the difference between them.

The two stars with the greatest differences in the estimation of temperature are stars with peculiarities in the spectra. One of the cases where we failed to determine gravity is a Cepheid. For estimating the metallicity, we failed in the case of two close binaries, an RS CVn-like variable and a Cepheid (HD 101602, both gravity and metallicity were estimated wrong for this variable star). 


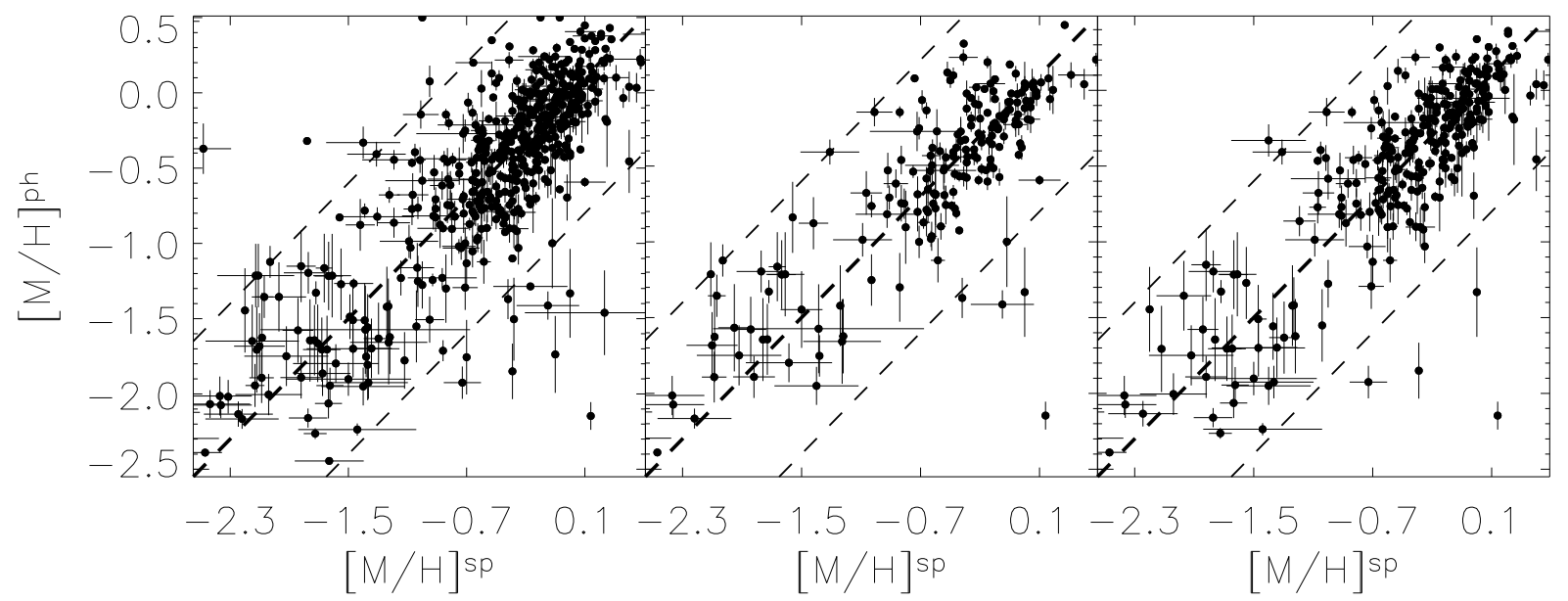

Fig. 17. The test of the method on the calibration sample for estimating metallicity. In the left panel there are all stars with $\sigma_{[\mathrm{M} / \mathrm{H}]}<0.3$ for the estimated metallicity and photometric errors better than 0.05 mag in all colors. The middle panel shows a subsample of the left panel falling into the most suitable region on the $\left(\log T_{\text {eff }},[\mathrm{M} / \mathrm{H}]\right)$ plane as described in the text, and in the right panel, we plot a subsample of the left panel with $A_{V}[\mathrm{mag}] / d[\mathrm{kpc}] \in[0.1,2.0]$. The dashed lines are the median line and $[\mathrm{M} / \mathrm{H}]^{s p}=[\mathrm{M} / \mathrm{H}]^{\mathrm{ph}}+0.9,[\mathrm{M} / \mathrm{H}]^{s p}=[\mathrm{M} / \mathrm{H}]^{\mathrm{ph}}-0.9$ lines.
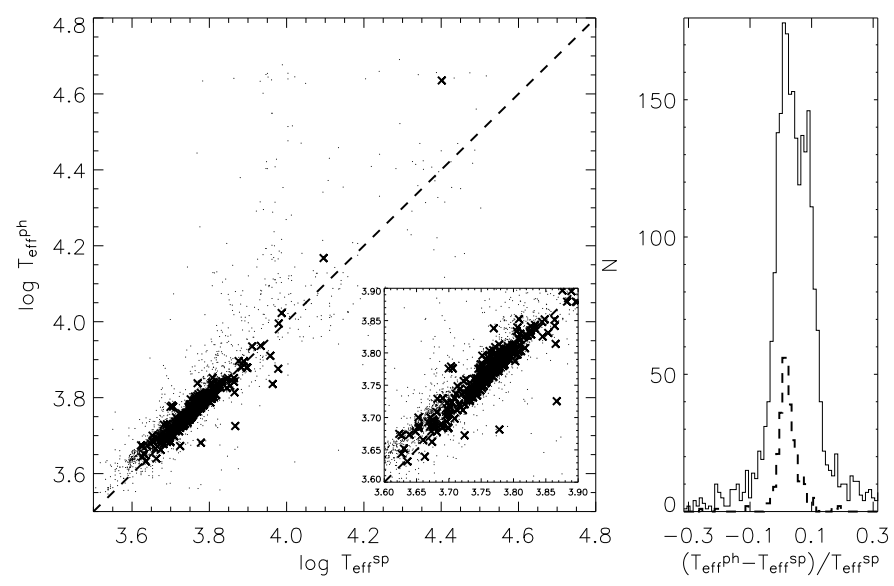

Fig. 18. Comparison between the temperature determined from photometry (our method) and spectroscopy (compiled catalog). Dots are all stars independent of the weight of the solution and the rms-error of the estimated temperature, crosses are stars with a weight of the solution $w>0.8$, photometric errors better than $0.05 \mathrm{mag}$ in all bands, $\sigma_{\log T_{\mathrm{eff}}^{\mathrm{ph}}}<0.05$ and $A_{V}[\mathrm{mag}] / d[\mathrm{kpc}] \in[0.1,2.0]$ (criterion $1 \& 3$ as described in Sect. 4.3.2). The right panel shows the corresponding histograms for both subsamples.

There is only one star for which the reason for the failure is unclear: BD +30 2611. This star is a suspected Hipparcos binary star, although the radial velocity measurements did not prove the binarity (Sperauskas \& Bartkevicius 2002).

In all cases of failure, the stars in question did not satisfy the assumptions underlying our classification procedure. Binaries and variables cannot be estimated using the synthetic grid of models for "normal" single stars. Exotic stars (from the point of view of chemical abundance) like carbon stars were rejected at the very first step of the method by the method itself, which failed to find satisfying synthetic grid points. The same happens if we try to use photometric observations of a galaxy as an input (we used the catalog of Gavazzi \& Boselli 1996). In the case of binaries and variable stars we may get a wrong estimation of the parameters, if we have no idea about the true nature of the star and if we have no information about the distance of the star and the extinction.
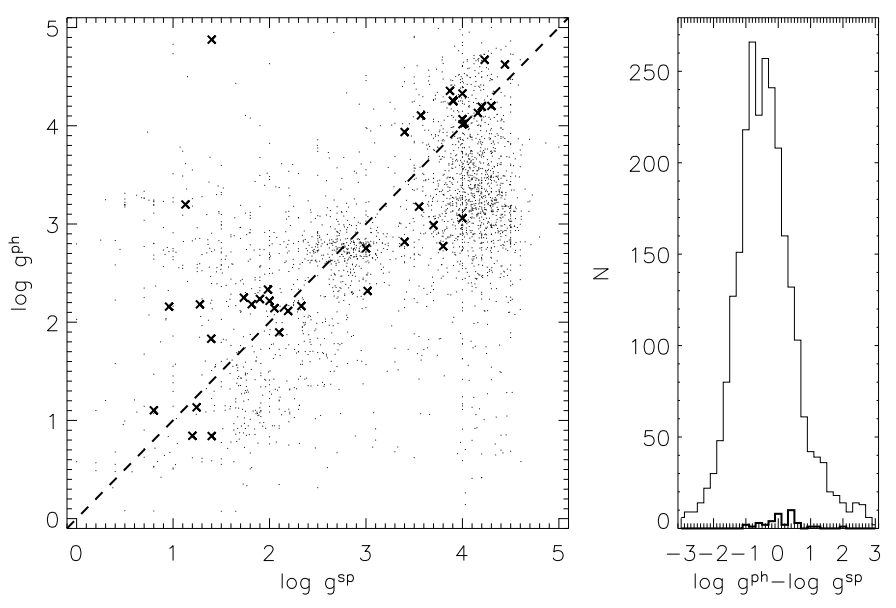

Fig. 19. Comparison between gravities determined from photometry (our method) and spectroscopy (compiled catalog). Dots are all stars independent of the weight of the solution and the rms-error of the estimated gravity, crosses are stars with a weight of the solution $w>0.8$, photometric errors better than 0.05 mag in all bands, $\sigma_{\log g^{\text {ph }}}<0.5$ and $A_{V}[\mathrm{mag}] / d[\mathrm{kpc}] \in[0.1,2.0]$ (criterion $1 \& 3$ as described in Sect. 4.3.2). The right panel shows the corresponding histograms for both subsamples.

\section{Results and conclusions}

We developed and tested a new method determining the astrophysical parameters from broad-band photometry. This method does not rely on a predefined model for the stellar population, but requires assuming the law for the extinction in the direction of the estimated star. In principle, the extinction law can be determined by the method itself with the use of stars with a unique solution for their astrophysical parameters, which is independent of the size of extinction.

We used an interval-cluster analysis as the mathematical core for the method. For a given input photometric system (here Johnson $B, V$, and 2MASS $J, H$, and $K_{\mathrm{S}}$ ), this method allows us to select the regions in the parameter space where a (unique) solution is possible and to identify regions in the parameter space where no solutions are possible due to the complicated topology of the color and parameter spaces. The astrophysical parameters determined with the use of this method are defined in the system 

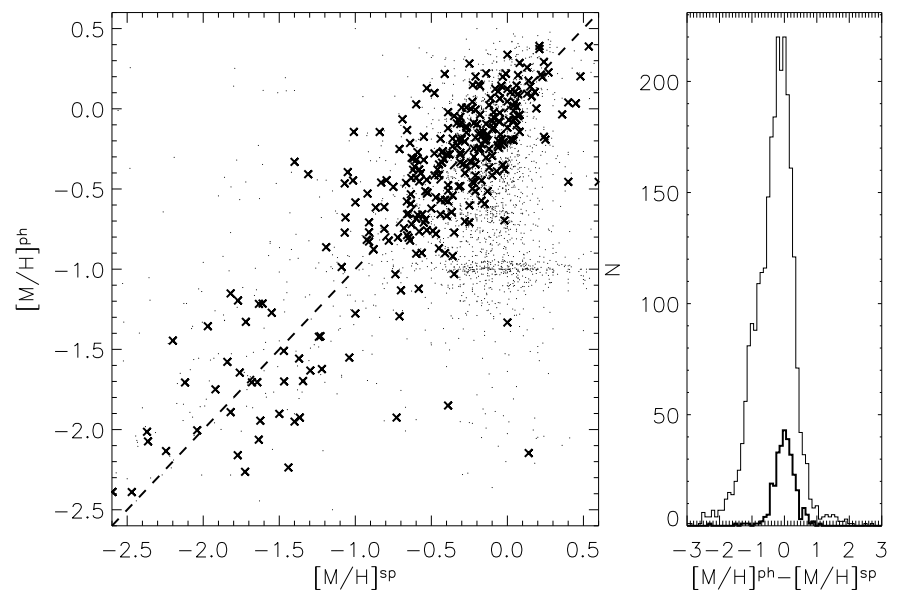

Fig. 20. Comparison between metallicities determined from photometry (our method) and spectroscopy (compiled catalog). Dots are all stars independent of the weight of the solution and the rms-error of the estimated metallicity, crosses are stars with a weight of the solution $w>0.8$, photometric errors better than $0.05 \mathrm{mag}$ in all bands, $\sigma_{[\mathrm{M} / \mathrm{H}]}<0.3$ and $A_{V}[\mathrm{mag}] / d[\mathrm{kpc}] \in[0.1,2.0]$ (criterion $1 \& 3$ as described in Sect. 4.3.2). The right panel shows the corresponding histograms for both subsamples.

Table 4. Reason for the failure of the parameter estimation.

\begin{tabular}{|c|c|c|c|}
\hline \multicolumn{4}{|c|}{$\log T_{\text {eff }}$} \\
\hline name & $\log T_{\mathrm{eff}}^{\mathrm{sp}}$ & $\log T_{\mathrm{eff}}^{\mathrm{ph}}$ & \\
\hline HD 18078 & 4.003 & 4.654 & A0p \\
\hline HD 96446 & 4.401 & 4.635 & B2IIIp \\
\hline \multicolumn{4}{|c|}{$\log g$} \\
\hline name & $\log g^{\mathrm{SP}}$ & $\log g^{\mathrm{ph}}$ & \\
\hline $\mathrm{BD}+302611$ & 1.13 & 3.20 & \\
\hline HD 101602 & 1.40 & 4.88 & $\begin{array}{c}\text { UZ Cen, } \\
\text { Cep-type variable }\end{array}$ \\
\hline \multicolumn{4}{|c|}{$[\mathrm{M} / \mathrm{H}]$} \\
\hline name & {$[\mathrm{M} / \mathrm{H}]^{\mathrm{sp}}$} & {$[\mathrm{M} / \mathrm{H}]^{\mathrm{ph}}$} & \\
\hline $\mathrm{BD}+302611$ & -1.40 & -0.33 & \\
\hline HD 101602 & 0.14 & -2.15 & $\begin{array}{c}\text { UZ Cen, } \\
\text { Cep-type variable }\end{array}$ \\
\hline HD 154338 & 0.0 & -1.33 & $\begin{array}{c}\text { V991 Sco, } \\
\text { RS CVn-type variable }\end{array}$ \\
\hline HD 3940 & -0.39 & -1.85 & $\begin{array}{l}\text { V755 Cas, } \\
\text { Algol-type }\end{array}$ \\
\hline HD 6582 & -0.73 & -1.93 & SB \\
\hline
\end{tabular}

of the selected models (synthetic or empirical) and suffer from all the systematic errors which are inherent in these models.

We have compiled a catalog of stars with astrophysical parameters known from spectroscopic observations to calibrate the Kurucz models and the estimated parameters.

In the Johnson $B, V$, and 2MASS $J, H$, and $K_{\mathrm{S}}$ photometric system and with mean errors of input colors better than $0.01 \mathrm{mag}$ the best results are achieved for main sequence G-K stars (most preferable region, $\mathrm{G} 6$ to $\mathrm{K} 9$ ). In this case the parameters are accurate to $\sigma_{\log T_{\text {eff }}}<0.05, \sigma_{\log g}<0.3$, and $\sigma_{[\mathrm{M} / \mathrm{H}]}<0.3$. If the accuracy of the color measurements drops to $0.05 \mathrm{mag}$, the most preferable region with the accuracy defined above $\left(\sigma_{\log T_{\text {eff }}}<0.05, \sigma_{\log g}<0.3\right.$, and $\left.\sigma_{[\mathrm{M} / \mathrm{H}]}<0.3\right)$ shrinks to main sequence K6-K9 stars; meanwhile, the accuracy for the rest of the most preferable region drops dramatically, especially for gravity. Nevertheless, even for $0.05 \mathrm{mag}$ the accuracy of the determined parameters for the rest of most preferable regions (main sequence G6 to K5) stays within $\sigma_{\log T_{\text {eff }}}<0.1$, $\sigma_{\log g}<2.0$, and $\sigma_{[\mathrm{M} / \mathrm{H}]}<0.4$. The definition of "the most preferable regions" depends on the combination of photometric bands, desired precision for the result and the precision of the input photometry.

We find that the biggest problem in determining astrophysical parameters is not to determine a solution but to distinguish between possible solutions that correspond to the input colors. We propose and tested a simple criterion for the selection of a solution based on the estimated extinction. Final tests with the compiled catalog proves the reliability and effectiveness of the method by comparing spectroscopic (literature) and photometric (our method) determinations of parameters.

The proposed method was initially designed to search for sparse stellar groups in deep sky surveys, but it also suits the study of populations in the Galaxy without a predefined model for the distribution of stars. It is possible to use the method to determine an extinction law.

Acknowledgements. This research was partially performed when ANB was at the Astronomisches Rechen-Institut, Heidelberg, where he gratefully acknowledges the DFG grant RO 528/9-1. A.N.B. is grateful to Dr. Castelli for help with ATLAS9 program. This research made use of the SIMBAD database, operated at the CDS, Strasbourg, France.

\section{References}

Borkova, T. V., \& Marsakov, V. A. 2005, Astron. Rep., 49, 405 Buser, R. 1978, A\&A, 62, 411

Cardelli, J. A., Clayton, C., \& Mathis, J. S. 1989, ApJ, 345, 245

Castelli, F., \& Kurucz, R. L. 2003, http: //kurucz . harvard.edu, ODFNEW models with $l_{\mathrm{p}}=1.25$, in Modelling of Stellar Atmospheres, ed. N. Piskunov, W. W. Weiss, \& D. F. Gray (ASP), IAU Symp., 210, A20

Cayrel de Strobel, G., Soubiran, C., Friel, E. D., Ralite, N., \& Francois, P. 1997, Ap\&SS, 124, 299

Carpenter, J. M. 2001, AJ, 121, 2851

Cutri, R. M., Skrutskie, M. F., Van Dyk, S., et al. 2006, Explanatory Supplement to the 2MASS All Sky Data Release, http://www.ipac.caltech.edu/ 2mass/

D’Antona, F., \& Mazzitelli, I. 1994, APJS, 90, 467

Erspamer, D., \& North, P. 2003, A\&A, 398, 1121

Gavazzi, G., \& Boselli, A. 1996, Astrophys. Lett. Comm., 35, 1

Girardi, L., Bertelli, G., Bressan, A., et al. 2002, A\&A, 391, 195

Grebel, E. K., \& Roberts, W. J. 1995, ApJS, 109, 293

Gustafsson, B., Edvardsson, B., Eriksson, K., et al. 2003, in Modelling of Stellar Atmospheres, ed. N. Piskunov, W. W. Weiss, \& D. F. Gray (ASP), IAU Symp., 210, A4

Heiter, U., Kupka, F., van't Veer-Menneret, et al. 2002, A\&A, 392, 619

Johnson, H. L., \& Morgan, W. W. 1953, ApJ, 117, 313

Kharchenko, N. V. 2001, Kinematics and Physics of Celestial Bodies, 17, 409

Koen, C., Marang, F., Kilkenny, D., \& Jacobs, C. 2007, MNRAS, 380, 1433

Lenz, D. D., Newberg, Jo, Rosner, R., et al. 1998, ApJS, 119, 121

Lejeune, T., Cuisinier, F., \& Buser, R. 1997, Ap\&SS, 125, 229

Martins, L. P., \& Coelho, P. 2007, MNRAS, 381, 1329

Materne, J. 1997, ApJ, 74, 235

Nordström, B., Mayor, M., Andersen, J., et al. 2004, A\&A, 418, 989

Palla, F., \& Stahler, S. W. 1993, ApJ, 418, 414

Samet, H. 1994, The Design and Analysis of Spatial Data Structures (AddisonWesley, Reading)

Schaller, G., Shaerer, D., Meynet, G., \& Maeder, A. 1992, ApJS, 96, 269

Silverman, B. W. 1986, Density Estimation for Statistics and Data Analysis (London: Chapman \& Hall)

Schmidt-Kaler, T. 1982, Landolt-Börnstein Numerical Data and Functional Relationships in Science and Technology. New Series, Group IV (New York, Berlin-Heidelberg: Springer-Verlag Press), 4, 31

Scott, D. W., \& Sain, S. R. 2004, Multi-Dimensional Density Estimation, in Handbook of Statistics, 23, Data Mining and Computational Statistics, ed. C. R. Rao, \& E. J. Wegman (Amsterdam: Elsevier)

Schlegel, D. J., Finkbeiner, D. P., \& Davis, M. 1998, ApJ, 500, 525

Smith, K. C., \& Dworetsky, M. M. 1993, A\&A, 274, 335

Sperauskas, J., \& Bartkevicius, A. 2002, Astron. Nachr., 323, 139

Soubiran, C., Katz, D., \& Cayrel, R. 1998, Ap\&SS, 133, 221

Straižys, V. 1977, Multicolor Stellar Photometry (Vilnius: Mokslas Pub.)

Straižys, V., Liubertas, R., \& Valiauga, G. 1997, Baltic Astron., 6, 601

Takeda, Y., Ohkubo, M., Sato, B., Kambe, E., \& Sadakane, K. 2005, Publ. Astron. Soc. Jap., 57, 27 